(2) Open Access Full Text Article

ORIGINALRESEARCH

\title{
Network Pharmacology Analysis of the Mechanisms of Compound Herba Sarcandrae (Fufang Zhongjiefeng) Aerosol in Chronic Pharyngitis
}

\section{Treatment}

\author{
Yanping Zhang ${ }^{1}, *$ \\ Taohua Yuan $\mathbb{D}^{2, *}$ \\ Yunsong $\mathrm{Li}^{\prime}$ \\ Ning $\mathrm{Wu}^{2}$ \\ Xiaotian Dai $\mathbb{D}^{3}$ \\ 'Guizhou University of Traditional \\ Chinese Medicine, Guiyang, Guizhou, \\ People's Republic of China; ${ }^{2}$ Guizhou \\ Medical University, Guiyang, Guizhou, \\ People's Republic of China; ${ }^{3}$ Department \\ of Mathematics and Statistics, University \\ of Calgary, Calgary, Alberta, Canada
}

*These authors contributed equally to this work
Correspondence: Ning Wu

Guizhou Medical University, Guiyang,

Guizhou, 550025, People's Republic of

China

Tel/Fax +86 I3765080008

Email1638210715@qq.com

Xiaotian Dai

Department of Mathematics and

Statistics, University of Calgary, Calgary,

AB, T2N IN4, Canada

Tel/Fax + I 4357544980

Email xiaotian.dai@ucalgary.ca
Purpose: This study aimed to investigate the molecular mechanisms of compound herba Sarcandrae aerosol, also known as the Fufang Zhongjiefeng (FFZJF) aerosol, in treating chronic pharyngitis (CP) using network pharmacology and in vivo experimental approaches. Methods: Active compounds and putative targets of five herbs in FFZJF were identified from the Traditional Chinese Medicine Systems Pharmacology Database and Analysis Platform, Chemistry Database, and Swiss Target Prediction databases. The therapeutic targets of CP were obtained from OMIM, Durgbank, DisGeNT, and GAD databases. The active compounds-target networks were constructed using Cytoscape 3.6.1. The overlapping targets of FFZJF active compounds and CP targets were further analyzed using the String database to construct protein-protein interaction (PPI) network. KEGG pathway and Gene Ontology enrichment analysis was performed using the Database for Annotation, Visualization, and Integrated Discovery. The predicted targets and pathways were validated in a group A $\beta$-hemolytic streptococcus-induced rat CP model.

Results: There were 45 active compounds identified from FFZJF and 11 potential protein targets identified for CP treatment. PPI network demonstrated that IL6, PTGS2, TLR-4, and TNF may serve as the key targets of FFZJF for the treatment of CP. The main functional pathways involving these key targets include cytokine secretion, inflammatory response, MyD88-dependent toll-like receptor signaling pathway, toll-like receptor signaling pathway, TNF signaling pathway, and NF- $\mathrm{kB}$ signaling pathway. In a rat $\mathrm{CP}$ model, the elevation of serum TNF- $\alpha$, IL1 $\beta$, and IL6 levels, as well as the upregulation of TLR-4, MyD88, NF- $\kappa B$ P65 in the pharyngeal mucosal tissues could be effectively reduced by FFZJF treatment in a dose-dependent manner.

Conclusion: Through a network pharmacology approach and animal study, we predicted and validated the active compounds of FFZJF and their potential targets for CP treatment. The results suggest that FFZJF can markedly alleviate GAS-induced chronic pharyngitis by modulating the TLR-4/MyD88/NF- $\mathrm{BB}$ signaling pathways.

Keywords: chronic pharyngitis, Fufang Zhongjiefeng Aerosol, FFZJF, network pharmacology, TLR-4/MyD88/NF- $\kappa$ B signaling pathway

\section{Introduction}

Chronic pharyngitis (CP) is a diffusive inflammation of the pharyngeal mucosa, submucosa, and pharyngeal lymphoid tissues, with a high incidence that accounts for approximately $10-20 \%$ of throat diseases. ${ }^{1,2}$ According to the epidemiological 
data, at least $20 \%$ of the adult population suffers from chronic pharyngitis worldwide. ${ }^{3}$ The symptoms of CP are stubborn, and the disease is difficult to cure and causes great inconvenience to the patient. At present, well-known international medical treatments are lozenges, laser and cryotherapy, and antibiotics. However, the disease is likely to break out repeatedly. In recent years, the abuse of many antibiotic drugs leads to the increased the risk of antibiotic resistance and the risk of adverse reactions ${ }^{4}$ as well as a significant decrease of the curative effects. Group A $\beta$-hemolytic Streptococcus (GAS)-induced pharyngitis is often considered as a trigger of $\mathrm{CP}$, and in $56-97 \%$ of $\mathrm{CP}$ cases, GAS pharyngitis precedes lesion formation. ${ }^{5}$

CP recurs easily, often has a prolonged disease course, and is difficult to treat. ${ }^{6}$ The causes of CP can be infectious (bacterial or viral), non-infectious (excessive alcohol use, smoking, voice abuse or misuse), ${ }^{7}$ or a recurring acute pharyngitis that is not being treated properly. ${ }^{7-11}$ Among the bacterial CP, GAS bacterium is the most common pathogen associated with the disease. ${ }^{7,12,13}$ Compound Herba Sarcandrae aerosol, also known as the Fufang Zhongjiefeng (FFZJF) aerosol, has been shown to be clinically effective in treating $\mathrm{CP}$ and it has been approved by Guizhou Medical Products Administration for human use. A previous study reported that FFZJF has antiinflammatory and anti-bacterial properties with small side effects, in vitro and in animals, ${ }^{14}$ long-term use, but its exact mechanism remains unclear. Traditional Chinese medicine (TCM) has a long history of viewing an individual or patient as a system with different statuses and has accumulated numerous herbal formulae. The holistic philosophy of TCM shares much with the key ideas of emerging network pharmacology and network biology, and meets the requirements of overcoming complex diseases. ${ }^{15,16}$ Network pharmacology is a technique used to systematically evaluate the pharmacological effects of drugs with multiple components and multiple targets by establishing links between targets, drugs, and diseases based on the principles of systems biology, which shares a similar holistic philosophy to traditional Chinese medicine (TCM) ${ }^{15,16}$ Therefore, network pharmacology is an important tool to analyze the effective components and therapeutic target of TCM. ${ }^{17}$

In this study, we used a network pharmacology approach to determine the effective ingredients, key targets, and related signaling pathways of FFZJF for the treatment of CP. A rat bacterial CP model $^{5}$ was then established to evaluate the pharmacodynamic effects of
FFZJF and to verify the molecular targets and signaling pathways predicted by network pharmacology. The results of this study will aid further investigation of the underlying mechanism of FFZJF in treating chronic pharyngitis.

\section{Materials and Methods}

Experimental Animal, Drugs and Reagents

Sixty specific-pathogen-free Sprague Dawley rats, including 30 females and 30 males, weighing 210-230 g, were purchased from Qinglongshan Experimental Animal Farm (Nanjing, Jiangsu Province, China) [License number: SCXK (Su) 2017-0001]. FFZJF was prepared using Zhongjiefeng (Herba Sarcandrae), Maidong (Ophiopogonis Radix), Honghua (Carthami Flos), Yuxingcao (Houttuyniae Herba), and Jiegeng (Platycodonis Radix) at a 4:3:2:1:1 ratio. The authenticity of herbs was validated by Deputy Chief Pharmacist Xianmei Xu (Guiyang College of Traditional Chinese Medicine Second Affiliated Hospital). The FFZJF aerosol was prepared by the hospital laboratory (Qian Yao Z20140036, equivalent to $2 \mathrm{~g}$ raw herbs per $\mathrm{mL}$ aerosol preparation). Ampicillin Sodium for Injection (AS) (Guo Yao H13020426) was purchased from North China Pharmaceutical (Shijiazhuang City, Hebei Province, China). Group A $\beta$-hemolytic Streptococcus (GAS, Cat\# FSCC225016) was purchased from Guangzhou Huankai Bio-Technology.SYBR ${ }^{\circledR}$ Premix Ex Taq (RR820A), PrimeScript $^{\mathrm{TM}}$ RT reagent Kit with gDNA Eraser (RR047A), RNAiso Plus (9108), all purchased from Takara Japan; DEPC (D105557, Aladdin Biochemistry in Shanghai); TLR-4 Antibody (GR163524-6, British Abcam Corporation), MyD88 Antibody (GR142247-2,British Abcam Corporation), NF-кB P65 Antibody (00057397, American Proteintech Corporation); TLR-4, MyD88, NF$\kappa B$ P65 PCR primers (Synthesis of Nanjing Kingsley Biotechnology Co., Ltd). TNF- $\alpha$, IL1 $\beta$, and IL6 ELISA Kit (CSB-E11987r, CSB-E08055r, CSB-E04640r, Cusabio).

\section{Identification of FFZJF Active Compounds and Putative Compound Targets}

The active compounds in FFZJF were collected from the TCM Systems Pharmacology Database and Analysis Platform (TCMSP; https://old.tcmsp-e.com/tcmsp.php) ${ }^{18}$ and Chemistry Database (http://www.organchem.csdb.cn/ scdb/default.htm?nCount $=13303008) .{ }^{19}$ The absorption, distribution, metabolism, and excretion (ADME) profiles of these compounds were obtained via literature searches and used to facilitate the screening of effective 
ingredients. ${ }^{20}$ The pharmacokinetics and pharmacodynamics criteria included oral bioavailability $(\mathrm{OB}) \geq 30 \%$, drug-likeness $(\mathrm{DL}) \geq 0.18$, and half-life (HL) $\geq 4 \mathrm{~h}$ (The main screening conditions of drug components were certain OB and DL levels. If one component does not meet one of the two conditions, it is still retained if it has good DL and HL levels or activities). ${ }^{21,22}$ The corresponding targets of the putative compounds were searched in the TCMSP and the Swiss Target Prediction database (http:// www.swisstargetprediction.ch/). ${ }^{15,23}$ To expand the potential targets, the molecular similarity match tool, the Simplified Molecular Input Line Entry System (SMILES) in PubChem (https://pubchem.ncbi.nlm.nih.gov/\#query= Nevadensia), ${ }^{23}$ was used. These two groups of targets were entered into the UniProt database with the organism defined as "Homo sapiens" and were considered FFZJF potential targets. UniProt (https://www.uniprot.org), ${ }^{23}$ is used for finding gene names and UniProt numbers of the putative target genes. $^{24}$

\section{Identification of Chronic Pharyngitis-Associated Targets}

Potential target genes of $\mathrm{CP}$ were collected from Online Mendelian Inheritance in Man (OMIM) (https://omim. org/), ${ }^{25}$ DrugBank Online (https://www.drugbank.ca/), ${ }^{26}$ DisGeNT database (https://www.disgenet.org/), ${ }^{26}$ and Genetic Association Database (GAD) (https://geneticasso ciationdb.nih.gov/ $/)^{25}$ with the keyword of "chronic pharyngitis". The overlapping and repeat targets were excluded. UniProt (https://www.uniprot.org) was used for unifying gene names and UniProt numbers.

\section{Network Construction and Pathway Enrichment}

After the putative targets of the FFZJF active compounds and the known therapeutic targets of $\mathrm{CP}$ were identified as described above, the protein-protein interaction (PPI) analysis was performed using the Search Tool for the Retrieval of Interacting Genes (String, Version 10.5, https://string-db.org/) database. ${ }^{27}$ A Venn diagram (http:// bioinformatics.psb.ugent.be/webtools/Venn//) ${ }^{25}$ was then produced to identify the intersection nodes of FFZJF and chronic pharyngitis.

The FFZJF-active compound-CP target network and herbs-active ingredient network were then constructed and visualized using Cytoscape software (version 3.6.1). ${ }^{27}$ Gene ontology (GO) annotation and Kyoto Encyclopedia of Genes and Genomes (KEGG) pathway enrichment analysis were performed to predict gene functions using Database for Annotation, Visualization and Integrated Discovery (DAVID) version 6.8 (https://david.ncifcrf. gov/). ${ }^{27}$ The GO describes three distinct aspects gene functions in terms of biological process, cellular component, and molecular function. $P$-value $<0.05$ was set as the cut-off criteria for significantly enriched KEGG pathways. The top key pathways related to FFZJF treatment of CP were selected based on the count values.

\section{Animal Model and Drug Treatment}

Experimental procedures used in this study followed the procedures described previously. ${ }^{28,29}$ All experimental procedures involving animals were approved by the Institutional Animal Care and Use Committee (IACUC) (Ethical number:1709051). After one-week acclimation, the 60 rats were randomly divided into two groups. The first cohort $(n=10)$ received no drug intervention and were used as blank control group. The other 50 rats received pharyngeal inoculation of $10^{12} \mathrm{CFU} / \mathrm{mL}$ GAS to establish a CP model. After inoculation, the bacteria were allowed to colonize for 15 days. The general health conditions of the animals were monitored daily. Five rats died due to the improper operations of a laboratory staff during the creation of the animal model. On Day 16, 3 rats were randomly selected and euthanized, and the pharyngeal mucosal tissues were collected for histopathological examination. The establishment of the GAS CP rat model was confirmed according to the symptoms, signs, and histopathological examination. The rats with confirmed CP were randomly divided into the control model group, ampicillin sodium group (AS), FFZJF high-dose (FFZJFH), FFZJF medium-dose (FFZJF-M), and FFZJF low-dose (FFZJF-L).

According to the literature, ${ }^{30}$ the rats in the FFZJF-L, FFZJF-M, and FFZJF-H group received 2.33, 4.66, and $9.32 \mathrm{~g} / \mathrm{kg}$ FFZJF in $20 \mathrm{~mL}$ saline nebulization, respectively. The rats in the control and treated CP model groups received an equal volume of saline nebulization once daily for 14 consecutive days as a control treatment. The rats in the AS group received a daily intraperitoneal injection of ampicillin sodium at a dose of $0.93 \mathrm{~g} / \mathrm{kg}$ for 14 days. At $24 \mathrm{~h}$ after the last treatment, the animals were euthanized and the serum and pharyngeal mucosal tissues were collected for further analysis. Half of each tissue sample was fixed in $4 \%$ paraformaldehyde, and the other half was frozen and stored at $-80^{\circ} \mathrm{C}$. 


\section{Measurement of the Serum Content of TNF- $\alpha$, ILI $\beta$, and IL6 Protein}

24 hours after the final medication, several steps are taken: 1) Anesthesia with $10 \%$ chloral hydrate solution $\mathrm{mL} / 100 \mathrm{~g}$ intraperitoneal injection; 2) taking blood from the abdominal aorta; 3) after $2 \mathrm{~h}$ at room temperature, $3000 \mathrm{r} / \mathrm{min}, 4^{\circ} \mathrm{C}$ Centrifugation (radius $13.5 \mathrm{~cm}$ ) for $10 \mathrm{~min}$; 4) take the supernatant and keep it in $-80^{\circ} \mathrm{C}$ Refrigerator; 5) serum TNF- $\alpha$, IL1 $\beta$, IL6 levels in rats were measured by ELISA method. The operation was carried out strictly according to the instructions of the kit. TNF- $\alpha$, IL1 $\beta$, IL6 levels in serum were calculated. ${ }^{31}$

\section{Measurements of mRNA Expression} Levels of TLR-4, MyD88, and NF-kB P65 in Pharyngeal Mucosa Tissue by Quantitative Reverse TranscriptionPolymerase Chain Reaction (qRT-PCR)

The mRNA levels of TLR-4, MyD88, and NF-кB P65 in the pharyngeal mucosal tissue were determined using a qRT-PCR. In brief, total RNA was extracted using the Trizol reagent and cDNA was prepared. SYBR $^{\circledR}$ Premix Ex Taq, PrimeScript ${ }^{\mathrm{TM}}$ RT reagent Kit with gDNA Eraser, and RNAiso Plus were purchased from Takara (Kusatsu, Shiga, Japan). qRT-PCR was performed in a $20 \mu \mathrm{L}$ reaction volume using the following conditions: pre-denaturation at $95^{\circ} \mathrm{C}$ for 10 min, followed by 40 cycles of denaturation at $95^{\circ} \mathrm{C}$ for $5 \mathrm{~s}$ and annealing/extension at $60^{\circ} \mathrm{C}$ for $1 \mathrm{~min}$. StepOnePlus ${ }^{\mathrm{TM}}$ Real-Time PCR System (Applied Biosystems, Foster City, CA, USA) was used for qRTPCR. The primers were synthesized by GenScript Biotechnology (Nanjing, Jiangsu Province, China) and their sequences are shown in Table 1. The expression levels of TLR-4, MyD88, and NF-кB P65 mRNAs were normalized to GAPDH and calculated using the $2^{-\Delta \Delta \mathrm{Ct}}$ method.

\section{Histological Examination of Pharyngeal Mucosal Tissue and Immunohistochemical (IHC) Staining}

Serial sections $(4 \mu \mathrm{m})$ were obtained from paraformaldehyde-fixed, paraffin-embedded rat pharyngeal mucosal tissues for histological analysis and IHC. The pharyngeal mucosal tissue morphology and anatomy were examined and compared using hematoxylin and eosin (HE) staining. ${ }^{32}$

For IHC, after antigen retrieval, the endogenous peroxidase activity was quenched, and the nonspecific antibody binding was blocked. The tissue sections were then incubated at $4{ }^{\circ} \mathrm{C}$ overnight with specific primary antibodies (at 1:200 dilution), followed by incubation with the corresponding secondary antibodies (at 1:200 dilution) at $4{ }^{\circ} \mathrm{C}$ for $50 \mathrm{~min}$. Mouse anti-TLR-4 (lot GR163524-6) and rabbit anti-MyD88 (lot GR1422472) antibodies were purchased from Abcam (Shanghai City, China), and the rabbit anti-NF-kB P65 (lot 00057397) antibody was from Proteintech (Wuhan

Table I List of Primer Sequences Used for RT-PCR Analysis in This Study

\begin{tabular}{|c|c|c|c|c|c|}
\hline $\begin{array}{l}\text { Gene } \\
\text { Name }\end{array}$ & Sequence of Primer & $\begin{array}{l}\text { Fragment } \\
\text { Length (bp) }\end{array}$ & $\begin{array}{l}\text { Annealing } \\
\text { Temperature }\left({ }^{\circ} \mathrm{C}\right)\end{array}$ & $\begin{array}{l}\text { Accession } \\
\text { Numbers }(\mu \mathrm{L})\end{array}$ & $\begin{array}{l}\text { Average } \\
\text { Efficiencies }\end{array}$ \\
\hline TLR-4-F & $\begin{array}{l}\text { 5'-AGGGTTTCCTGTCAGTATCAAGT } \\
\text { TT-3' }\end{array}$ & 310 & $60^{\circ} \mathrm{C}$ & 0.4 & $99 \%$ \\
\hline TLR-4-R & 5'-TGATGCCTCCCTGGCTCCT-3' & 310 & $60^{\circ} \mathrm{C}$ & 0.4 & \\
\hline MyD88-F & 5'-AAGGGTATGGAGAACATGAA-3' & 367 & $60^{\circ} \mathrm{C}$ & 0.4 & $98 \%$ \\
\hline MyD88-R & 5'-TTTTCGATGCCTTTATCTGCT-3' & 367 & $60^{\circ} \mathrm{C}$ & 0.4 & \\
\hline$N F-\kappa B$ P65-F & $\begin{array}{l}\text { 5'-TTGCTCCAGGTCTCGCTTCTTC } \\
\text { ACA-3' }\end{array}$ & 342 & $60^{\circ} \mathrm{C}$ & 0.4 & $100 \%$ \\
\hline$N F-\kappa B$ P65-R & 5'-TCCTTTCAATGGACCAACTGAA-3' & 342 & $60^{\circ} \mathrm{C}$ & 0.4 & \\
\hline GAPDH-F & $\begin{array}{l}\text { 5'-CAGGGCTGCTTTTAACTCTGGTA } \\
\text { A-3' }\end{array}$ & 101 & $60^{\circ} \mathrm{C}$ & 0.4 & $100 \%$ \\
\hline GAPDH-R & $\begin{array}{l}\text { 5'-GGGTGGAATCATATTGGAACATG } \\
\text { T-3' }\end{array}$ & 101 & $60^{\circ} \mathrm{C}$ & 0.4 & \\
\hline
\end{tabular}


City, Hubei Province, China). Following the secondary antibody incubation and washing, the sections were developed with 3, 3', 4, 4'-biphenyltetramine tetrahydrochloride (DAB) and counterstained with hematoxylin. The positive staining was identified as brown color granules. The expression patterns and

Table 2 The Active Compounds from the 5 Herbs in FFZJF

\begin{tabular}{|c|c|c|c|c|c|}
\hline Herb Name & MOL ID & Molecule Name & OB (\%) & DL & HL \\
\hline \multirow[t]{7}{*}{ Zhongjiefeng } & MOL007747 & Chloranoside a_qt & 84.11 & 0.23 & 7.3 \\
\hline & MOL007742 & Istanbulin-A & 80.1 & 0.2 & 5.34 \\
\hline & MOL000098 & Quercetin & 46.43 & 0.28 & 14.4 \\
\hline & MOL004373 & Anhydroicaritin & 45.41 & 0.44 & 15.01 \\
\hline & MOL007743 & ZINC00391893 & 41.92 & 0.2 & 16.69 \\
\hline & MOL007744 & Chloranthalactone A & 41.72 & 0.18 & 7.3 \\
\hline & MOL004568 & Engelitin & 36.27 & 0.7 & 17.6 \\
\hline \multirow[t]{10}{*}{ Maidong } & MOL005885 & $\alpha$-patchoulene & 54.64 & 0.11 & 12.16 \\
\hline & MOL002573 & $\beta$-patchoulene & 50.69 & 0.11 & 14.26 \\
\hline & MOL004679 & Longifolene & 39.49 & 0.11 & 11.2 \\
\hline & MOL00I732 & Glycerol & 72.87 & 0.01 & 11.45 \\
\hline & MOL000449 & Stigmasterol & 43.83 & 0.76 & 5.57 \\
\hline & MOL000I7I & Guaiol & 38.77 & 0.09 & 6.2 \\
\hline & MOL000I30 & Camphor & 67.17 & 0.05 & 11.34 \\
\hline & MOL00I083 & $\alpha$-cyperene & 53.05 & 0.13 & 12.75 \\
\hline & MOL004786 & 4-terpineol & 40.75 & 0.03 & 10.94 \\
\hline & MOL004358 & Linalool & 58.18 & 0.02 & 11.22 \\
\hline \multirow[t]{15}{*}{ Honghua } & MOL0027I2 & 6-hydroxykaempferol & 62.13 & 0.27 & 14.29 \\
\hline & MOL002680 & Flavoxanthin & 60.41 & 0.56 & 16.38 \\
\hline & MOL000098 & Quercetin & 46.43 & 0.28 & 14.4 \\
\hline & MOL00272I & Quercetagetin & 45.01 & 0.31 & 13.82 \\
\hline & MOL000449 & Stigmasterol & 43.83 & 0.76 & 5.57 \\
\hline & MOL002695 & Lignan & 43.32 & 0.65 & 14.88 \\
\hline & MOL000422 & Kaempferol & 41.88 & 0.24 & 14.74 \\
\hline & MOL002776 & Baicalin & 40.12 & 0.75 & 17.36 \\
\hline & MOL000953 & CLR & 37.87 & 0.68 & 4.52 \\
\hline & MOL002773 & Beta-carotene & 37.18 & 0.58 & 4.36 \\
\hline & MOL00I77I & Poriferast-5-en-3beta-ol & 36.91 & 0.75 & 5.07 \\
\hline & MOL000358 & Beta-sitosterol & 36.91 & 0.75 & 5.36 \\
\hline & MOL002698 & Lupeol-palmitate & 33.98 & 0.32 & 10.21 \\
\hline & MOL0027I4 & Baicalein & 33.52 & 0.21 & 16.25 \\
\hline & MOL0027I9 & 6-hydroxynaringenin & 33.23 & 0.24 & 15.67 \\
\hline \multirow[t]{6}{*}{ Yuxingcao } & MOL000098 & Quercetin & 46.43 & 0.28 & 14.4 \\
\hline & MOL004355 & Spinasterol & 42.98 & 0.76 & 5.32 \\
\hline & MOL000422 & Kaempferol & 41.88 & 0.24 & 14.74 \\
\hline & MOL00385I & Isoramanone & 39.97 & 0.51 & 4.79 \\
\hline & MOL004350 & Ruvoside_qt & 36.12 & 0.76 & 7.21 \\
\hline & MOL004345 & I-methyl-2-nonacosyl-4-quinolone & 31.54 & 0.5 & 16.13 \\
\hline \multirow[t]{7}{*}{ Jiegeng } & MOL004580 & Cis-Dihydroquercetin & 66.44 & 0.27 & $|4.5|$ \\
\hline & MOL004355 & Spinasterol & 42.98 & 0.76 & 5.32 \\
\hline & MOL006070 & Robinin & 39.84 & 0.71 & 16.67 \\
\hline & MOL006026 & Dimethyl 2-O-methyl-3-O-a-D-glucopyranosyl platycogenate A & 39.21 & 0.25 & 5.04 \\
\hline & MOL000006 & luteolin & 36.16 & 0.25 & 15.94 \\
\hline & MOL002998 & $\mathrm{IPH}$ & 36.05 & 0.01 & 12.07 \\
\hline & MOL00I689 & Acacetin & 34.97 & 0.24 & 17.25 \\
\hline
\end{tabular}

Abbreviations: FFZJF, Fufang Zhongjiefeng; DL, drug-like properties; HL, drug half-life; OB, oral bioavailability. 


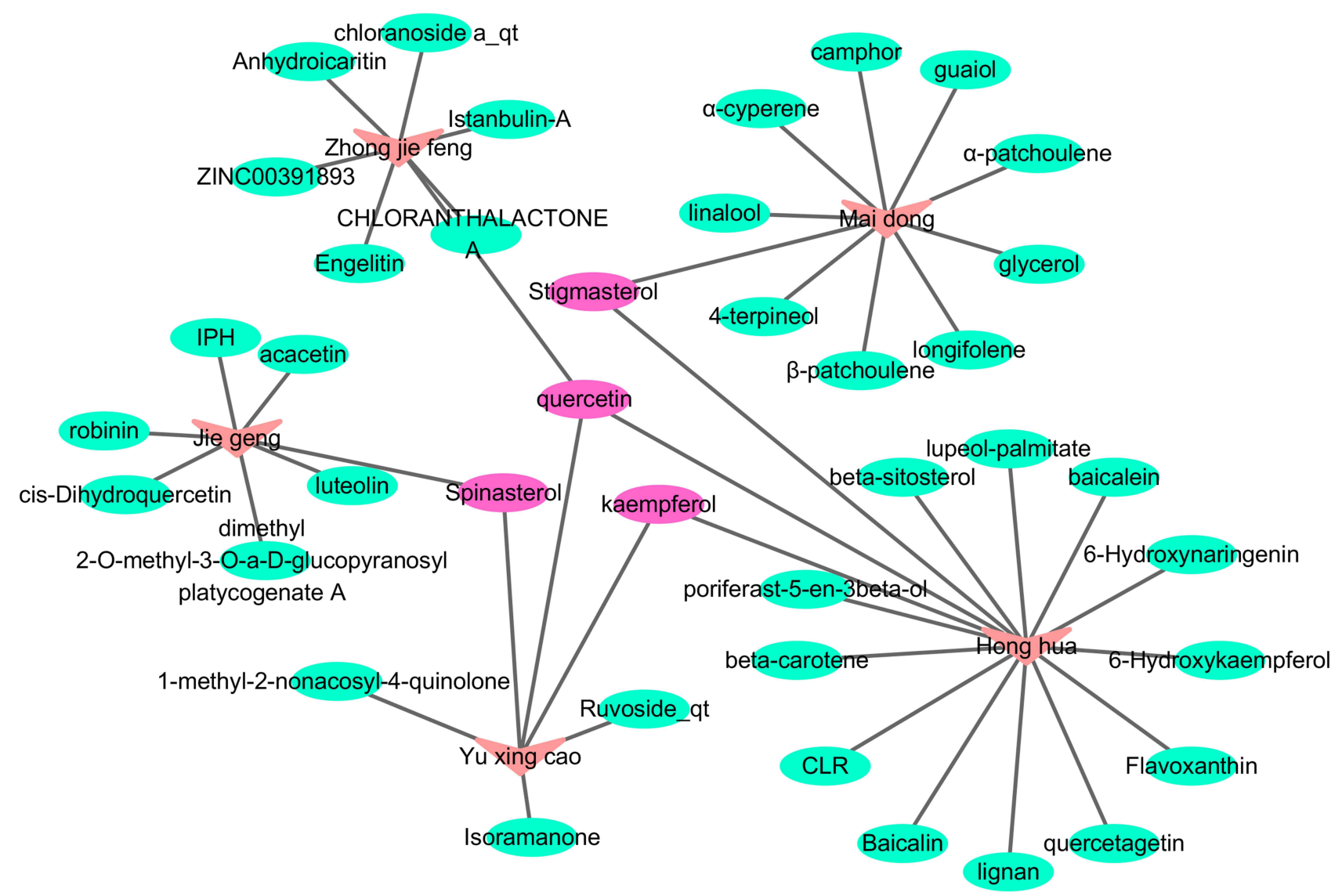

Figure I The network construction for herbs-active ingredient.

subcellular localization of the TLR-4, MyD88, and NF$\kappa \mathrm{B}$ P65 protein were examined. A Nikon H550S microscope (Nikon, Tokyo, Japan) was used to observe the staining. The relative expression levels of TLR-4, MyD88, and NF- $\kappa \mathrm{B}$ P65 proteins were quantitated using Integrated Optical Density (IOD) measured in Image-Pro plus 6.0 software and compared between the experimental groups.

\section{Statistical Analysis}

Statistical analyses were performed using SPSS software version 22.0 (Armonk city, New York state, USA). Unless otherwise noted, all data are presented as mean

Table 3 Information on Four Key Active Ingredients

\begin{tabular}{|l|l|l|l|}
\hline $\begin{array}{l}\text { Molecule } \\
\text { Name }\end{array}$ & Molecule ID & Degree & $\begin{array}{l}\text { Betweenness } \\
\text { Centrality }\end{array}$ \\
\hline Quercetin & MOL000098 & 3 & 0.42600423 \\
Spinasterol & MOL004355 & 2 & 0.27378436 \\
Stigmasterol & MOL000449 & 2 & 0.35940803 \\
Kaempferol & MOL000422 & 2 & 0.15221987 \\
\hline
\end{tabular}

\pm standard error of mean (SEM). The data were first subjected to the Shapiro-Wilk test for normality. Normally distributed data were analyzed using oneway analysis of variance (ANOVA), and the KruskalWallis test was used if the data did not pass the normality test. If the results from the ANOVA tests were statistically significant, the pair-wise comparisons were then performed. For pair-wise comparisons, the Fisher's least significant difference (LSD) tests were used.

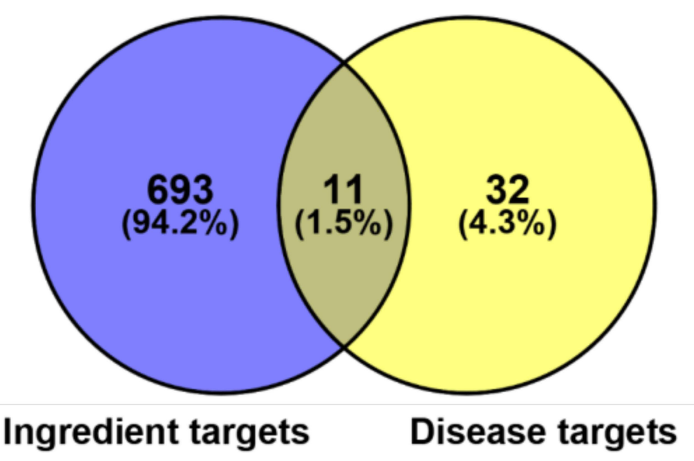

Figure 2 Venn diagram of FFZJF drug targets and CP-disease proteins. 
Comparisons with $p$ values $<0.05$ were considered statistically significant.

\section{Results}

\section{Active Compounds Identified in FFZJF and Their Putative Targets}

Database screening identified 189, 161, 200, 220, and 162 compounds in the five FFZJF herbal extracts, namely Zhongjiefeng, Maidong, Honghua, Yuxingcao, and Jiegeng, respectively. Using $\mathrm{OB} \geq 30 \%$, DL $\geq$ 0.18 , and half-life $\geq 4 \mathrm{~h}$ as the cut-off criteria, we identified 45 compounds from FFZJF, of which 7 from Zhong Jie Feng (15.6\%), 10 from Mai Dong (22.2\%), 15 from Hong hua, 6 from Yu Xing cao (33.3\%), and 7 from Jiegeng (15.6\%). According to relevant literature, ${ }^{21,22}$ the components with good retention activity but not satisfying the screening conditions were retained (Table 2). The network diagram of active components of Chinese herbal medicine was established
(Figure 1), Pink nodes represent herbs, Purple nodes represent key active ingredients after network analysis, and Green nodes represent other active ingredients. In the network diagram, the effective compounds were analyzed and arranged in descending order. The first four active compounds were quercetin, Spinasterol, Stigmasterol, kaempferol (Table 3). In addition, a total of 704 putative protein targets of these compounds were identified using the TCMSP and the Swiss Target Prediction databases.

\section{Prediction of FFZJF Molecular Targets for the Treatment of CP}

The CP-related target genes were screened from OMIM (https://omim.org/), DrugBank Online (https://www.drug bank.ca/), DisGeNT (https://www.disgenet.org/), and GAD (https://geneticassociationdb.nih.gov/). Using the keyword "chronic pharyngitis" to search the databases, 43 associated genes were identified. From the comparative analysis between the $43 \mathrm{CP}$-related and 704 putative

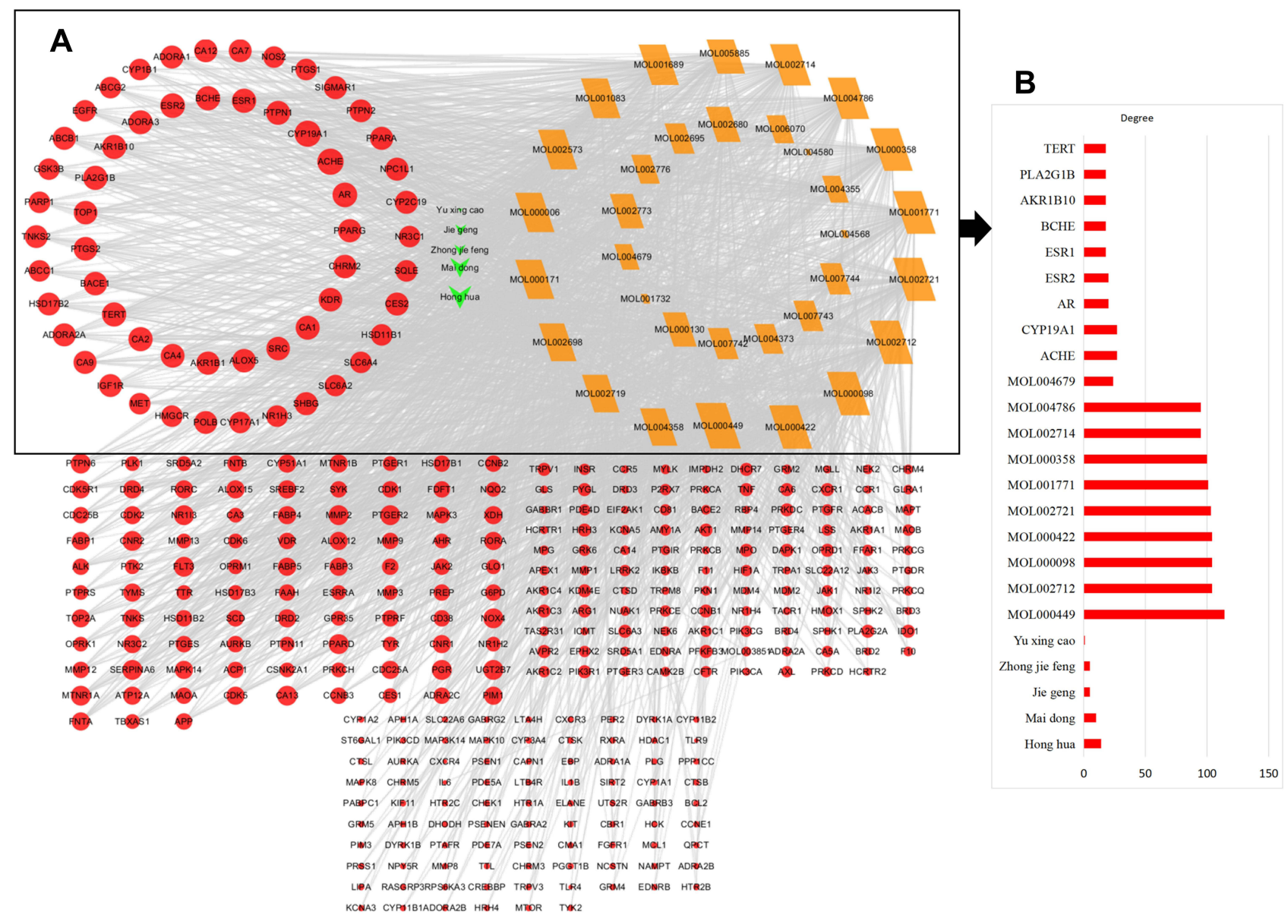

Figure 3 (A) Gene ontology analysis of key target genes. (B) Topology features of FFZJF-active compounds-CP network. 
FFZJF targets, 11 were predicted as FFZJF targets for the treatment of CP (Figure 2).

The network relationship between FFZJF, the active compounds, and CP targets are shown in Figure 3, which shows the interactions of targets containing 384 nodes and 2,252 edges (connecting lines) with an average node degree of 11.73 . The node size represented the degree value of the target. The shapes indicate nodes and the grey lines indicate interaction edges. The triangles and green colour indicate drugs. Diamond shapes and yellow colour indicate compounds. Circles and red colour indicate targets. According to the node degree, Honghua and Maidong were identified as the principle herbs. The top 10 predicted compounds and targets are shown in Table 4.

\section{Analysis of PPI}

The FFZJF-compounds-CP PPI network included 11 nodes and 37 edges (Figure 4). The larger the node and the darker the color was, the larger the degree value was. The larger the degree value was, the more important role

Table 4 Topology Features of FFZJF-Active Compounds-CP Network

\begin{tabular}{|l|l|l|l|}
\hline Name & Degree & $\begin{array}{l}\text { Betweenness } \\
\text { Centrality }\end{array}$ & $\begin{array}{l}\text { Closeness } \\
\text { Centrality }\end{array}$ \\
\hline Honghua & 14 & 0.008 & 0.413 \\
Maidong & 10 & 0.004 & 0.351 \\
Jiegeng & 5 & 0.001 & 0.300 \\
Zhongjiefeng & 5 & 0.001 & 0.290 \\
Yuxingcao & 1 & 0.000 & 0.244 \\
MOL000449 & 114 & 0.083 & 0.427 \\
MOL0027I2 & 104 & 0.051 & 0.417 \\
MOL000098 & 104 & 0.051 & 0.417 \\
MOL000422 & 104 & 0.055 & 0.421 \\
MOL00272I & 103 & 0.051 & 0.416 \\
MOL00I77I & 101 & 0.059 & 0.413 \\
MOL000358 & 100 & 0.056 & 0.412 \\
MOL0027I4 & 95 & 0.057 & 0.413 \\
MOL004786 & 95 & 0.084 & 0.409 \\
ACHE & 27 & 0.028 & 0.509 \\
CYPI9AI & 27 & 0.032 & 0.504 \\
MOL004679 & 24 & 0.002 & 0.356 \\
AR & 20 & 0.015 & 0.475 \\
ESR2 & 20 & 0.015 & 0.455 \\
ESRI & 18 & 0.011 & 0.453 \\
BCHE & 18 & 0.011 & 0.465 \\
AKRIBI0 & 18 & 0.011 & 0.441 \\
PLA2GIB & 18 & 0.015 & 0.479 \\
TERT & 18 & 0.012 & \\
\hline
\end{tabular}

Abbreviations: CP, chronic pharyngitis; FFZJF, Fufang Zhongjiefeng. the part played in the PPI. The average node degree was 6.73. Eight hub proteins, which were identified based on the node degree $>6.73$, ranked from the highest to the lowest degrees were IL6, PTGS2, TLR-4, TNF, ELANE, CXCR4, TLR9, and IL1B (Table 5). These targets are likely to be key targets for FFZJF treatment of CP, and TNF- $\alpha$, IL1 $\beta$, IL6, TLR-4 targets are selected for subsequent experimental verification.

\section{GO Enrichment and KEGG Pathway Analysis of the II Putative FFZJF Targets}

The 11 common targets shared by FFZJF active compound and $\mathrm{CP}$ disease-related proteins were further characterized for known functions using DAVID pathway analysis. GO enrichment was analyzed from the perspective of molecular function, biological process, and cellular component. Analyses using the GO database showed that the main enrichment of genes in the BP category was "inflammatory response", "positive regulation of NF-kappaB import into nucleus", "positive regulation of nitric oxide biosynthetic process", "positive regulation of interleukin-6 production", and "positive regulation of gene expression". The main MF categories were "heme binding", "cytokine activity", "enzyme binding”, "interleukin-1 receptor binding", "cytokine binding", "steroid hydroxylase activity", and "oxygen binding".The main CC categories were "cytoplasm", "cell surface", "extracellular region", "extracellular space",and "endoplasmic reticulum membrane" (Figure 5).

In addition, KEGG pathways of the target genes were analyzed and the top 15 of the 27 enriched pathways are shown in Figure 6. The 27 enriched pathways were found to be associated with viral infections, cancer, inflammation, and immunity. Among these pathways, the classic signaling pathways, including the toll-like receptor signaling pathway, TNF signaling pathway, NF- $\kappa \mathrm{B}$ signaling pathway, interactions between cytokine-cytokine receptors, NOD-like receptor signaling pathway, and HIF-1 signaling pathway were among the top 15 enriched with the most genes. In particular, the top-ranked Toll-like receptor signaling pathway, TNF signaling pathway, and NF- $\mathrm{BB}$ signaling pathway were enriched with genes such as IL6, TNF, TLR-4, and TLR9, these targets were tested and validated.

\section{General Health Conditions of the Animals in the Treatment Groups}

Four days after GAS inoculation, the animals started to show some symptoms, such as scratching in the mouth area, drinking more frequently, lethargy, fluffed coat, 


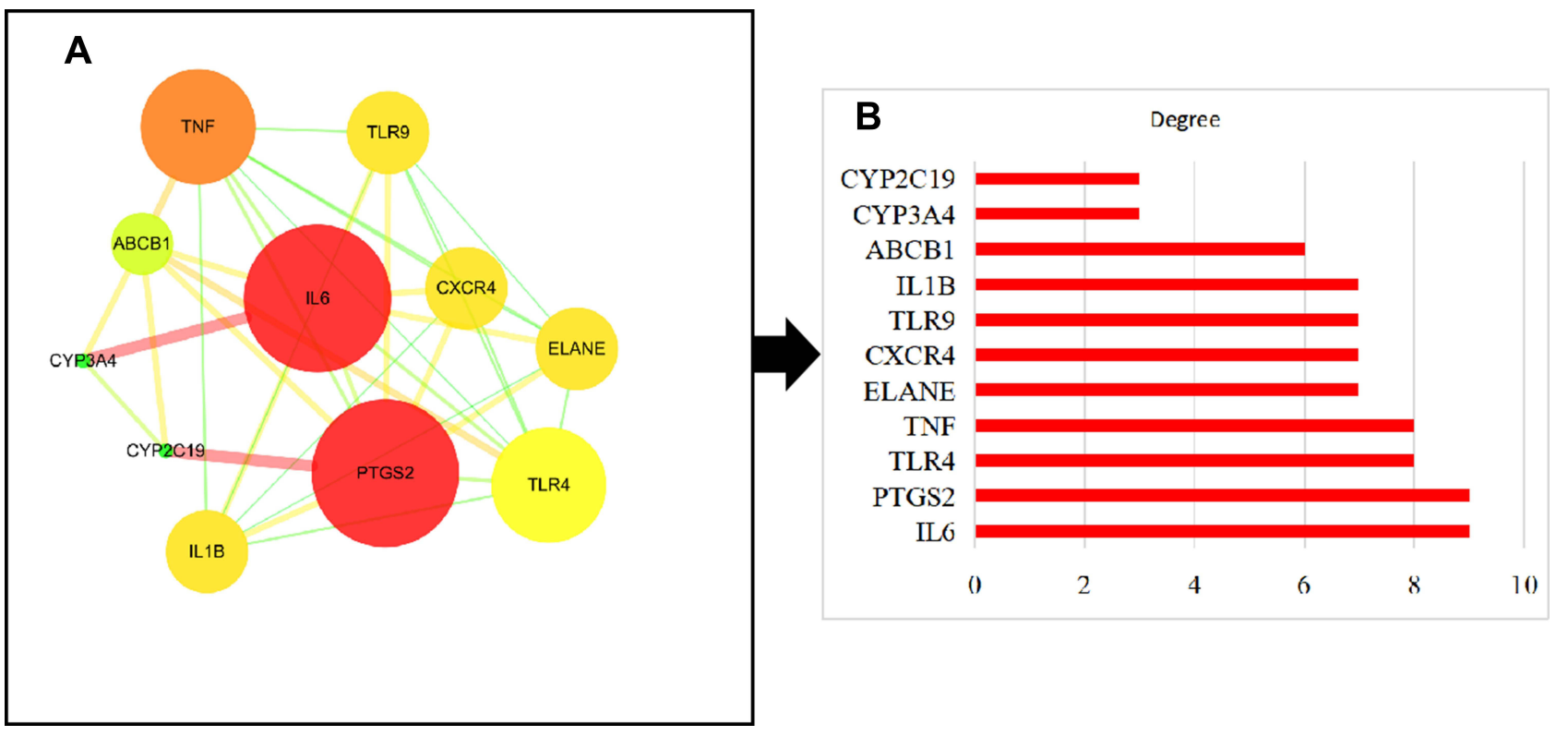

Figure 4 (A) PPI network of CP targets of FFZJF. (B) Hub genes of the PPI network.

and oral discharge (occasionally bloody). Some animals died during the $\mathrm{CP}$ model establishment. By day 16, oral discharge decreased gradually. However, the rats exhibited weight loss $(p<0.05)$ (Figure 7A), became less active, and consumed less food. Oral visual examination with a headlight showed chronic congestion in pharyngeal mucosal tissue, indicated by a dark redpurple colored, dry, dull, and swollen mucosa with some yellow discharge. After FFZJF or AS treatment, the overall health conditions of the animals markedly improved, as evidenced by the lustrous coat and increased food consumption, the weight increased $(p<0.05)$, but did not return to the weight of the rats

Table 5 Information on the Top-II Genes in PPI Network

\begin{tabular}{|l|l|l|l|}
\hline $\begin{array}{l}\text { Gene } \\
\text { Name }\end{array}$ & Degree & $\begin{array}{l}\text { Betweenness } \\
\text { Centrality }\end{array}$ & $\begin{array}{l}\text { Closeness } \\
\text { Centrality }\end{array}$ \\
\hline IL6 & 9 & 0.14 & 0.91 \\
PTGS2 & 9 & 0.14 & 0.91 \\
TLR4 & 8 & 0.02 & 0.83 \\
TNF & 8 & 0.02 & 0.83 \\
ELANE & 7 & 0.00 & 0.77 \\
CXCR4 & 7 & 0.00 & 0.77 \\
TLR9 & 7 & 0.00 & 0.77 \\
ILIB & 7 & 0.00 & 0.77 \\
ABCBI & 6 & 0.06 & 0.71 \\
CYP3A4 & 3 & 0.01 & 0.59 \\
CYP2CI9 & 3 & 0.01 & 0.59 \\
\hline
\end{tabular}

Abbreviation: PPI, protein-protein interaction. in the blank group $(p<0.05)$ (Figure 7B). Such improvement was particularly significant in the FFZJF-H and AS treatment groups. Moreover, oral examination showed overall normal pharyngeal mucosal tissue in the FFZJF-H and AS groups. In the FFZJF-L and FFZJF-M groups, the pharyngeal mucosal tissue showed a relatively normal color and morphology, except for some mild chronic congestion. The rats in the control group were healthy and had no anomalies throughout the study. The rats in the treated model group had CP symptoms and signs, which remained unrelieved throughout the study.

\section{Serum Levels of TNF- $\alpha$, ILI $\beta$, and IL6}

The treated model group had significantly elevated serum levels of TNF- $\alpha$, IL1 $\beta$, and IL6 compared to the non-CP control group $(p<0.01)$, which indicated the successful establishment of the CP model in vivo. Ampicillin sodium treatment significantly reduced the serum levels of TNF- $\alpha$, IL1 $\beta$, and IL6 as compared to the CP model group $(p<0.01)$. The animals treated with different doses of FFZJF showed decreased serum levels of TNF- $\alpha$, IL1 $\beta$, and IL6 at different degrees $(p<0.01)$, with FFZJF-H showing the greatest effect The curative effect of FFZJF-L group was the most obvious. Compared to the AS group, there was no significant difference in serum TNF- $\alpha$, IL1 $\beta$, and IL6 in FFZJF-H group $(p>0.05)$ (Table 6, Figure $8 \mathrm{~A}-\mathrm{C})$. 


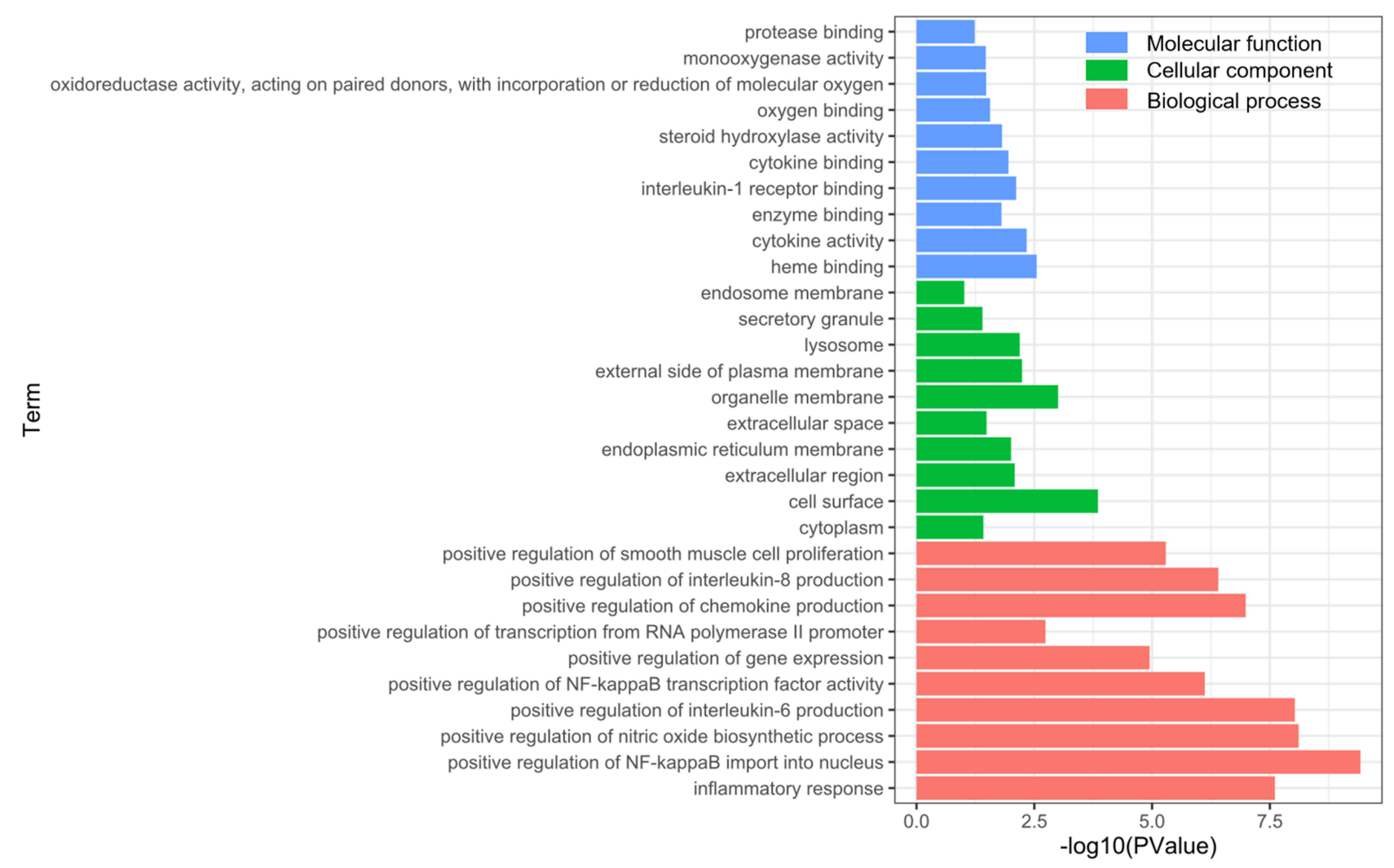

Figure 5 Gene ontology analysis of key target genes.

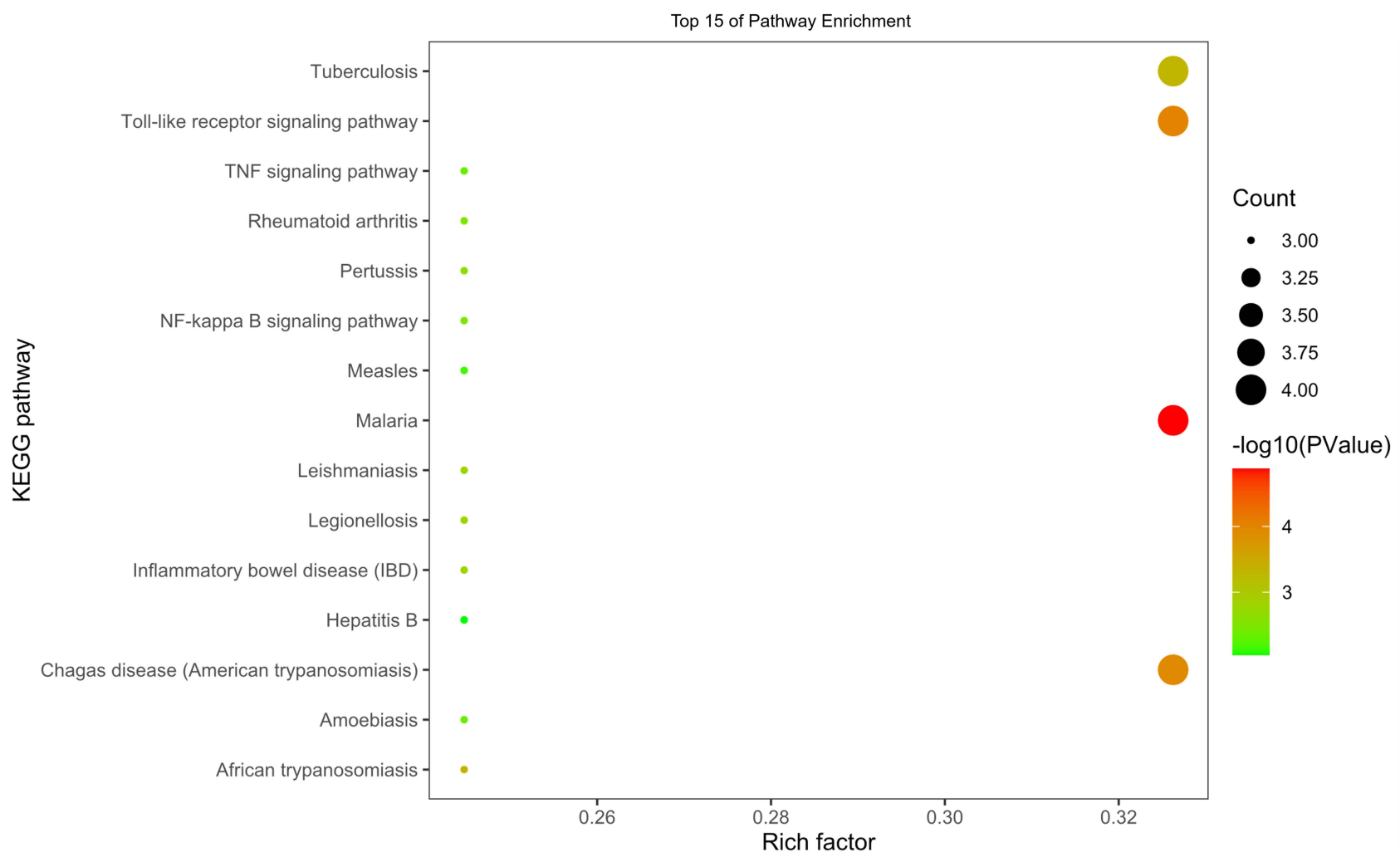

Figure 6 Top 15 pathways by KEGG analysis. 
A

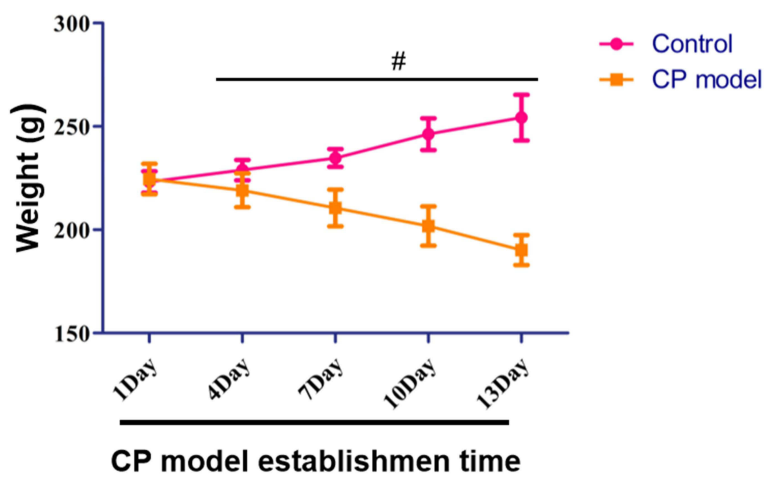

Figure 7 Changes of body weight of rats during modeling and administration.

\section{Histopathological Examination}

In the non-CP control group, the pharyngeal mucosal tissue exhibited normal microscopic anatomy. In the CP model group, submucosal edema with extensive inflammatory cell infiltration was observed, which also confirmed the successful establishment of CP model. The inflammatory cell infiltration reduced significantly in the AS group. In FFZJF groups, the alleviation of inflammatory cell infiltration increased as FFZJF dose increased, with the most significant reduction in the FFZJF-H group (Figure 9).

\section{The Expression of TLR-4, MyD88, and NF- $\kappa B$ P65}

The mRNA expression levels of TLR-4, MyD88, and NF- $\mathrm{B}$ P65 in pharyngeal mucosal tissues were measured using qRT-PCR and compared between the experimental groups. The $\mathrm{CP}$ model group had significantly increased mRNA levels of TLR-4, MyD88, and NF- $\mathrm{kB}$
B

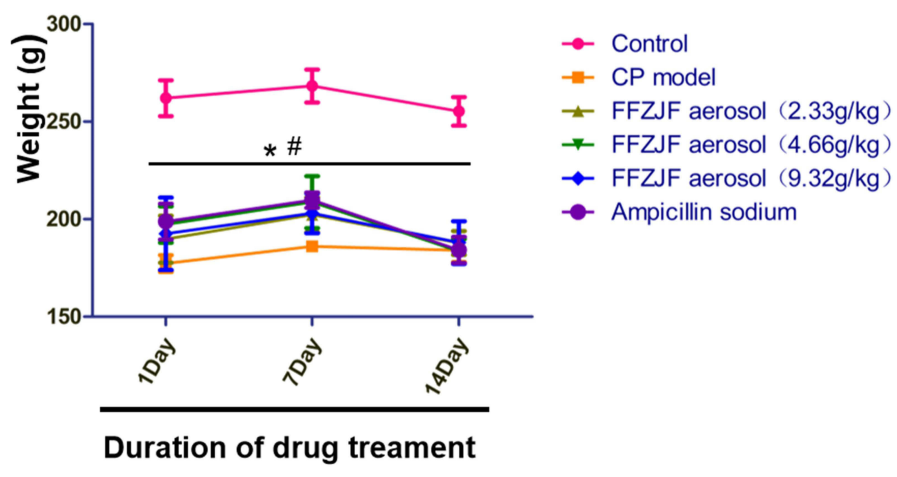

P65 in pharyngeal mucosa as compared with the no-CP control group $(p<0.01)$. Compared to the $\mathrm{CP}$ model group, the FFZJF-L group had a moderately reduced NF-кB P65 mRNA level $(p<0.05)$. The FFZJF-M group had a significant reduction in TLR-4 $(p<0.05)$,

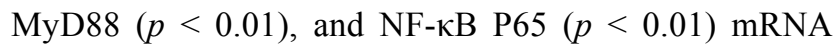
expression level, while the FFZJF-H and AS groups showed the greatest reduction in the expression of all three genes $(p<0.01)$ in their pharyngeal mucosal tissues.Compared with the AS group, the mRNA levels of TLR-4, MyD88 and NF-kB P65 in the FFZJF-H group were not statistically significant $(p>0.05)$ (Table 7,Figure 10A-C).

The protein expression levels of TLR-4, MyD88,

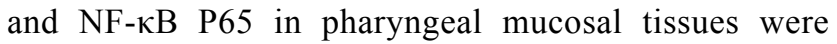
assessed using IHC and compared across the experimental groups. The subcellular location and tissue distribution of each protein were first evaluated. Positive TLR-4 staining was mainly associated with the cell membrane and cytosol of the lymphocytes, but the

Table 6 The Effect of FFZJF Aerosol on the Rat's Serum Levels of TNF- $\alpha$, ILI $\beta$, and IL6 (Mean \pm SEM, $n=8$ )

\begin{tabular}{|l|l|l|l|l|}
\hline Group & Dose $(\mathbf{g} / \mathbf{k g})$ & TNF- $\alpha$ & ILI $\beta$ & IL6 \\
\hline Control & - & $60.46 \pm 3.01$ & $453.24 \pm 35.71$ & $30.85 \pm 1.62$ \\
\hline CP model & - & $167.04 \pm 3.77^{* *}$ & $1733.48 \pm 71.92^{* *}$ & $98.39 \pm 3.43^{* *}$ \\
\hline FFZJF aerosol & 2.33 & $124.43 \pm 5.02^{* * \# \Delta}$ & $1400.86 \pm 73.97^{* * \# \Delta}$ & $78.62 \pm 2.29 * * \# \Delta$ \\
& 4.66 & $105.51 \pm 6.59^{* * \# \Delta}$ & $938.71 \pm 88.01^{\# \#}$ & $62.78 \pm 3.38^{* * \# \Delta}$ \\
& 9.32 & $74.87 \pm 3.29 * \# \#$ & $565.62 \pm 75.8 I^{\# \#}$ & $44.92 \pm 2.96 * * \#$ \\
\hline Ampicillin sodium & 0.93 & $88.29 \pm 3.48^{* * \#}$ & $581.81 \pm 49.49^{\# \#}$ & $42.93 \pm 3.8 I^{* * \#}$ \\
\hline
\end{tabular}

Notes: As compared to treated non-CP control group: ${ }^{*} p<0.05,{ }^{* *} p<0.01$; as compared to treated CP model group: ${ }^{\#} p<0.05$, ${ }^{\# \#} p<0.01$; as compared to treated Ampicillin sodium group: ${ }^{\Delta} P<0.05$.

Abbreviation: SEM, standard error of mean. 

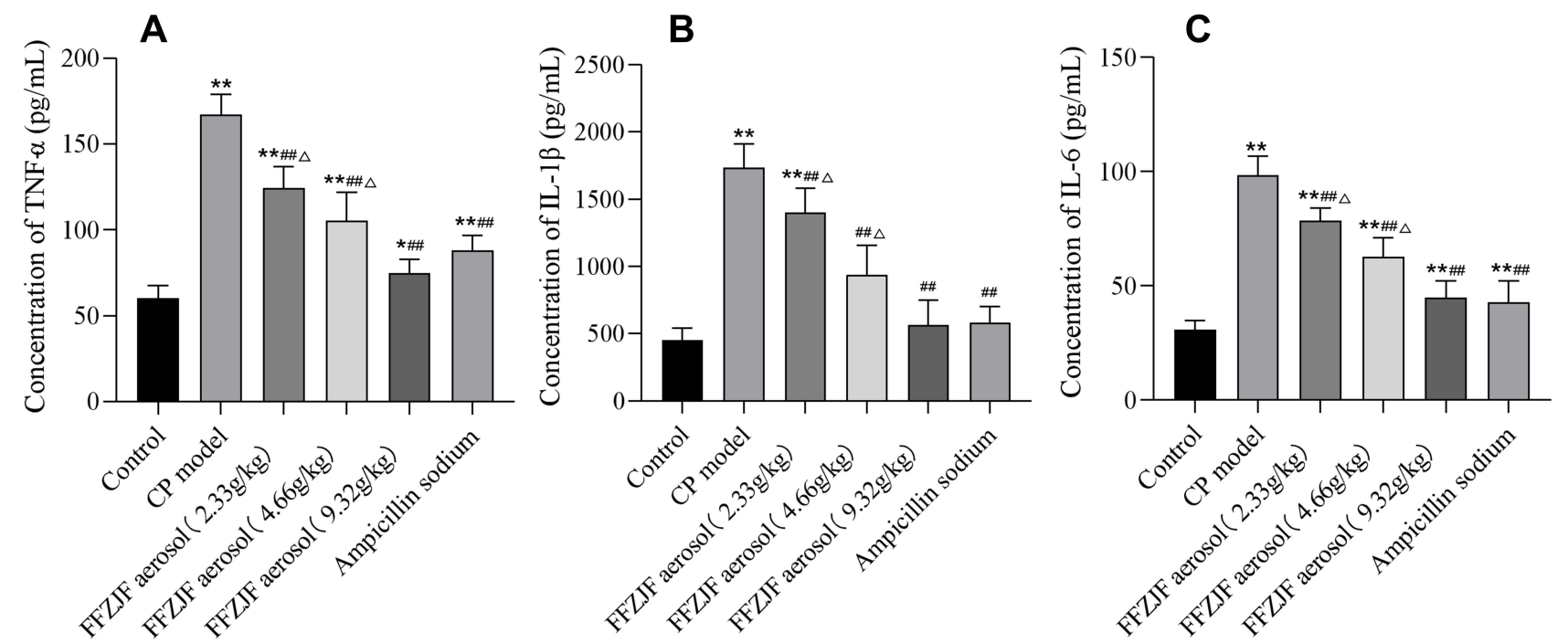

Figure 8 The effect of FFZJF aerosol on the rat's serum levels of TNF- $\alpha$, ILI $\beta$, and IL6 (Mean \pm SEM, $n=8)$. (A) TNF- $\alpha$; (B) ILI; (C) IL6.

Notes: As compared to treated non-CP control group: ${ }^{*} p<0.05,{ }^{*} p<0.01$; as compared to treated CP model group: ${ }^{\#} p<0.05$, ${ }^{\#} p<0.01$; as compared to treated Ampicillin sodium group: ${ }^{\Delta} \mathrm{P}<0.05$.

staining was relatively weak in other tissue types (Figure 11). MyD88 positive staining was mainly associated with the cytosol of all tissue and cell types

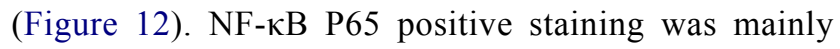
associated with the cytosol and nucleus in all tissue and cell types (Figure 13). Consistent with the mRNA expression data, the treated model group had elevated TLR-4, MyD88, and NF- $\kappa$ B P65 protein expression in the pharyngeal mucosal tissue compared to the non-CP controls $(p<0.01)$, which could be reduced effectively by AS $(p<0.01)$ or by FFZJF treatment at all dosages tested $(p<0.01)$, moreover, the effects of FFZJF on decreasing TLR-4,MyD88, and NF- $\kappa B$ P65 expression levels are dose-dependent. The higher the FFZJF concentration level is, the more obvious the treatment effects are. Compared to the AS group, the protein expressions of TLR-4, MyD88, and NF- $\mathrm{BB}$ P65 in FFZJF-L and FFZJF-M groups were significantly different $(P<0.05)$, and the protein expressions of TLR-4 in FFZJF-H group were significantly different $(P<$ $0.05)$, but there was no statistical significance observed in the expression levels of MyD88 and NF- $\mathrm{BB}$ P65 $(P>0.05)$ (Table 8, Figure 14).

\section{Discussion}

Chronic pharyngitis is a diffuse inflammation of the mucosa, submucosa, and lymphoid tissue of the pharynx. It has high prevalence, long duration of disease, easy recurrence, and a variety of symptoms. The main symptoms are sore throat, dry pharynx, itchy pharynx, foreign body sensation in pharynx, hyperemia and edema of pharyngeal mucosa, and hyperplasia of lymphoid follicles. The long-term use of antibiotics to treat bacterial chronic pharyngitis is likely to produce drug resistance and other side effects. ${ }^{7-11}$ In recent years, traditional Chinese medicine has achieved good results in the treatment of CP. FFZJF is a hospital preparation of Guizhou University of Traditional Chinese Medicine (Guizhou Medicines Z20140036). Prof. Zhang Yanping has optimized the prescription and dosage form of FFZJF according to the experience of Dr. Li Shengyue, a famous traditional Chinese medicine scientist. It has been used in clinic for more than ten years and has achieved satisfactory results. The prescription is composed of five components: Zhongjiefeng, Maidong, Honghua, Yuxingcao, and Jiegeng. It has the functions of clearing heat and detoxifying, reducing swelling and relieving pain, activating blood circulation, and promoting pharynx. It also has the characteristics of low price, rich drug source, convenient use form (atomizer), and small side effects, especially reducing the use of antibiotics. We also confirm that FFZJF single drug is nearly non-toxic. Among them, Honghua and Yuxingcao are well-known plants that are widely used as herbs as well as food. ${ }^{33-37}$ FFZJF has shown to be effective in terms of treating CP. ${ }^{11}$ More importantly, the application of FFZJF avoids the overuse of antibiotics in treating this chronic condition. However, the 


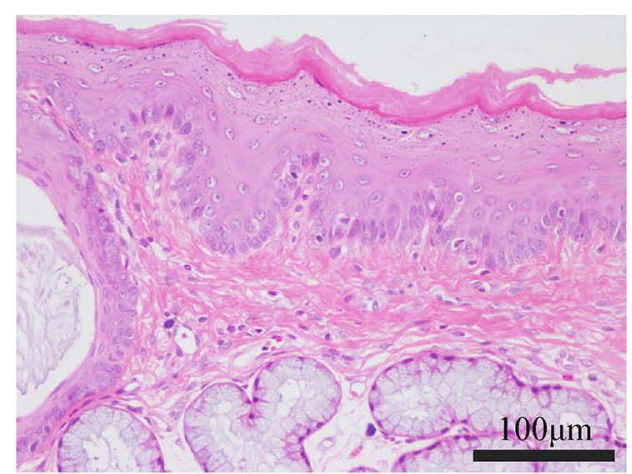

A

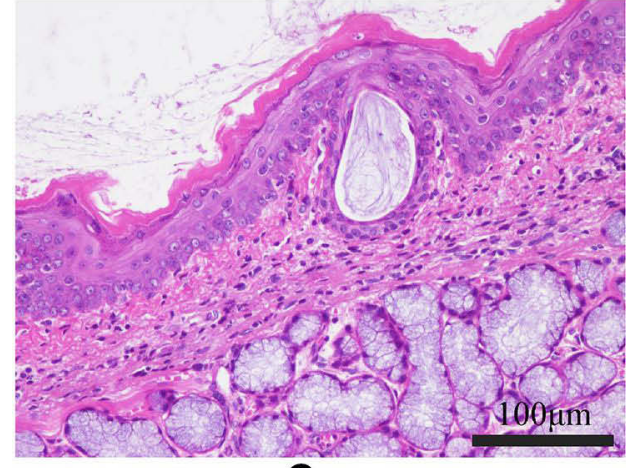

C

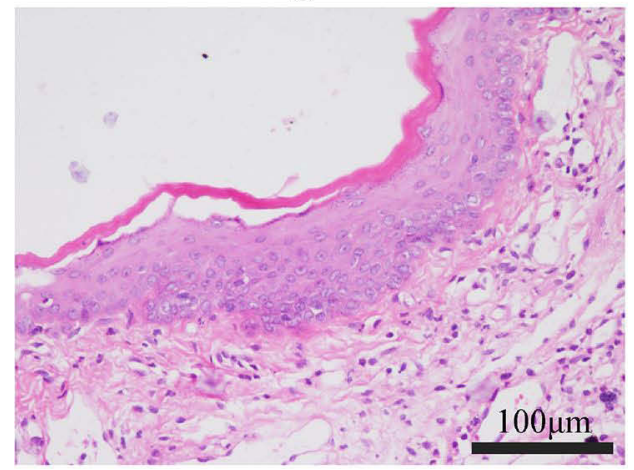

E

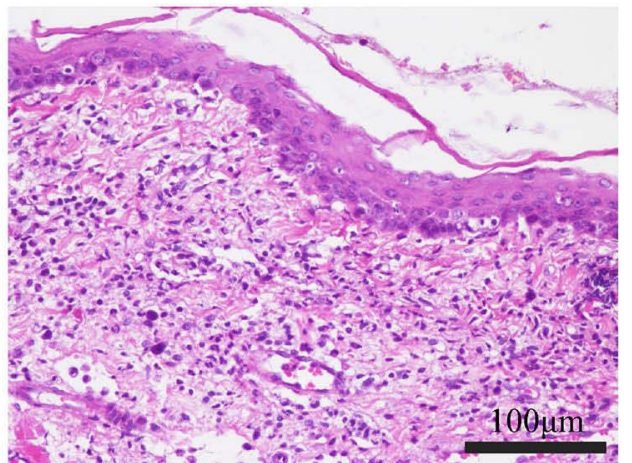

B

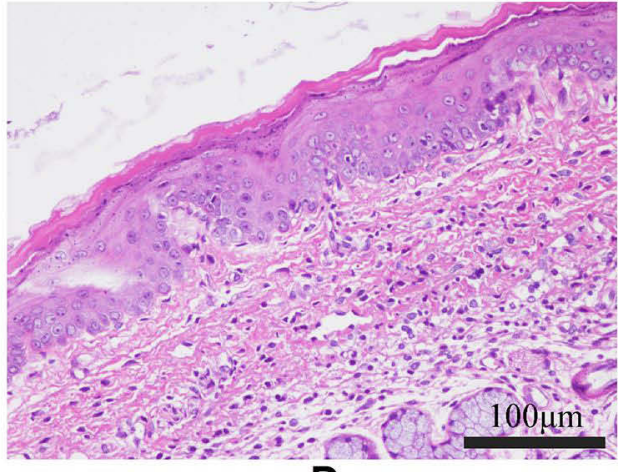

D

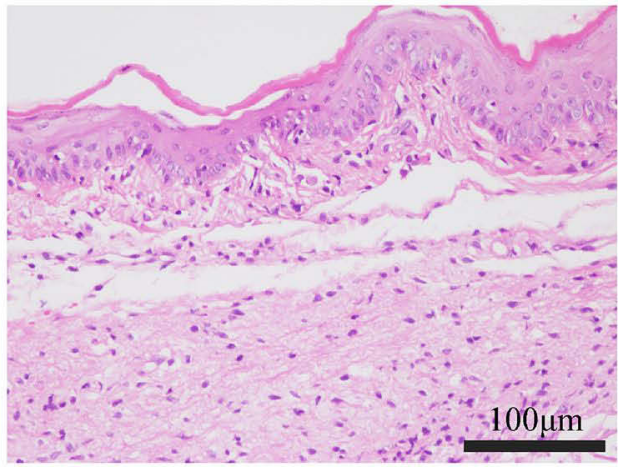

$\mathbf{F}$

Figure 9 Histopathological examinations of the pharyngeal mucosal tissue of the rats from each group (HE, 200x). (A) Control group; (B) Model group; (C) FFZJF-L group; (D) FFZJF-M group; (E) FFZJF-H group; (F) AS group. It can be seen that the morphology of the pharyngeal tissues in the control group remain intact based on microscopy. In the model group, the mucous membrane appears necrotic, the submucosal layer shows swelling (edema), and many inflammatory cells are present. The inflammation is significantly reduced in the FFZJF treatment groups. As the dose of FFZJF increases, the condition of inflammation improved.

mechanism of FFZJF has rarely been investigated. ${ }^{7-11}$ The network pharmacology is consistent with the holistic view of traditional Chinese medicine, such as the syndrome differentiation and treatment. ${ }^{15,16}$ Therefore, this study based on network pharmacology unveils the mechanism of FFZJF treatment of CP.

In this study, we used a network pharmacology approach combined with experimental validation to investigate the mechanisms of FFZJF for the treatment of CP. Our network pharmacology study identified 51 active compounds found in FFZJF. FFZJF-active compound-target $\mathrm{CP}$ network showed that the main active compounds included stigmasterol, 6-hydroxykaempferol, quercetin, kaempferol, and quercetagetin. Quercetin has good curative effects on pharyngitis, and kaempferol and quercetagetin have anti-inflammatory effects. $^{38,39}$ Meanwhile, the network suggests that the components Honghua, Maidong, Jiegeng, Zhongjiefeng play a key role in the treatment of $\mathrm{CP}$, which was confirmed by previous research. ${ }^{11}$ Zhongjiefeng, bitter, is the commonly used medicine for the treatment of throat diseases; Maidong, slightly bitter and slightly 
Table 7 The Effect of FFZJF Aerosol on the mRNA Levels of TLR-4, MyD88, and NF- $\kappa B$ P65 in the Rat's Pharyngeal Mucosal Tissues (Mean $\pm S E M, \mathrm{n}=8)$

\begin{tabular}{|l|l|l|l|l|}
\hline Group & Dose $(\mathbf{g} / \mathbf{k g})$ & TLR-4 & MyD88 & NF-kB P65 \\
\hline Control & - & $1 \pm 0.09$ & $1 \pm 0.04$ & $1 \pm 0.06$ \\
\hline CP model & - & $4.26 \pm 0.48^{* *}$ & $2.77 \pm 0.06^{* *}$ & $4.25 \pm 0.11^{* *}$ \\
\hline FFZJF aerosol & 2.33 & $3.23 \pm 0.34^{* * \Delta}$ & $2.44 \pm 0.11^{* * \Delta}$ & $3 \pm 0.29 * * \# \Delta$ \\
& 4.66 & $2.63 \pm 0.35^{* * * \Delta}$ & $1.95 \pm 0.12^{* * \# \Delta}$ & $2.2 \pm 0.09^{* * \# \Delta}$ \\
& 9.32 & $1.43 \pm 0.12^{\# \#}$ & $1.24 \pm 0.05^{\# \#}$ & $1.91 \pm 0.15^{* * \# \#}$ \\
\hline Ampicillin sodium & 0.93 & $1.31 \pm 0.14^{\# \#}$ & $1.21 \pm 0.17^{\# \#}$ & $1.61 \pm 0.17^{* * \# \#}$ \\
\hline
\end{tabular}

Notes: As compared to treated non-CP control group: ${ }^{*} p<0.05,{ }^{* *} p<0.01$; as compared to treated CP model group: ${ }^{\#} p<0.05$, ${ }^{*} p<0.01$; as compared to treated Ampicillin sodium group: ${ }^{\Delta} \mathrm{P}<0.05$.

cold, can moisturize lung and clear throat; Honghua can activate blood circulation and disperse blood stasis to relieve pain; Jiegeng can promote blood circulation and promote pharynx, detumescence, and pain relief. Modern pharmacological studies have shown that Zhongjiefeng and Maidong can reduce TNF- $\alpha$ and IL6 levels and inhibit the activation of transcription factor $\mathrm{NF}-\kappa \mathrm{B} .{ }^{40}$ The extraction of Yuxincao can downregulate the production of NO, TNF- $\alpha$ and interleukin in cells and inhibit the NF-KBp65 subunit of nuclear translocation. ${ }^{41}$ The active component of hydroxysafflor yellow A blocks I $\mathrm{B} B \alpha$ phosphorylation and degradation and prevents nuclear translocation. ${ }^{42}$ Platycodin-D can significantly inhibit IL1 $\beta$ induced NF- $\mathrm{BB}$ activation. ${ }^{43}$ Our previous study suggested that the treatment of gastroesophageal reflux pharyngitis symptoms and signs was improved by compound FFZJF aerosol and was better than that of some other widely-used medicines. ${ }^{44}$ Compound FFZJF aerosol can reduce the number of white blood cells, neutrophils and lymphocytes in plasma, and the level of serum IL-1. The lowest inhibitory concentration for streptococcus haemolyticus B in vitro was $105 \mathrm{mg} / \mathrm{mL}$, suggesting that compound FFZJF aerosol may be used to treat pharyngitis by antiinflammation and anti-bacterial, ${ }^{14}$ but the mechanism remains to be further studied. The PPI network revealed
A

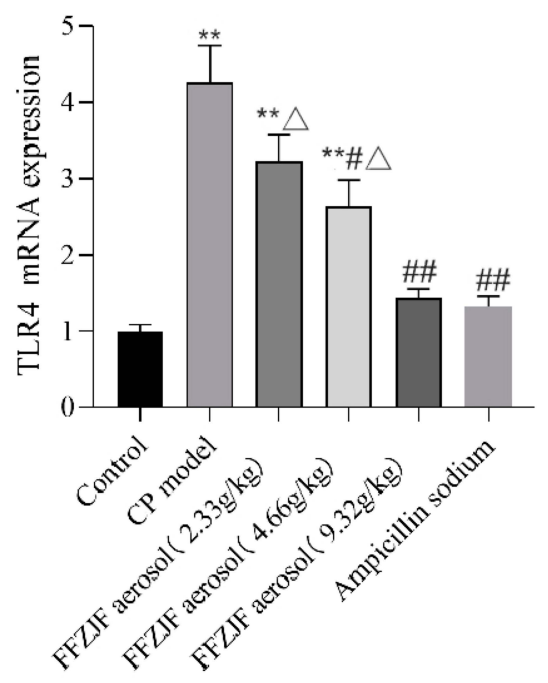

B

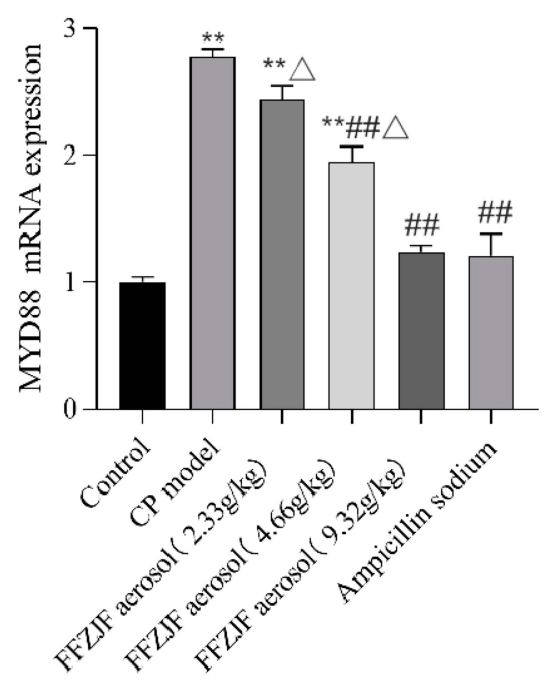

C

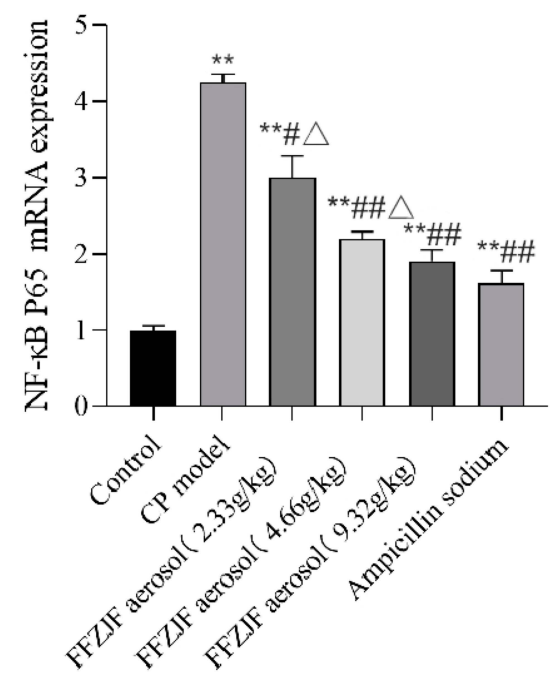

Figure 10 The effect of FFZJF aerosol on the mRNA levels of TLR-4, MyD88, and NF- $\kappa B$ P65 in the rat's pharyngeal mucosal tissues (Mean \pm SEM, $n=8)$. (A) TLR-4; (B) MyD88; (C) NF-KB P65.Note: As compared to treated non-CP control group: ${ }^{*} p<0.05,{ }^{* *} p<0.01$; as compared to treated CP model group: ${ }^{\#} p<0.05$, ${ }^{\# \#}<0.01$; as compared to treated Ampicillin sodium group: ${ }^{\Delta} \mathrm{P}<0.05$. 


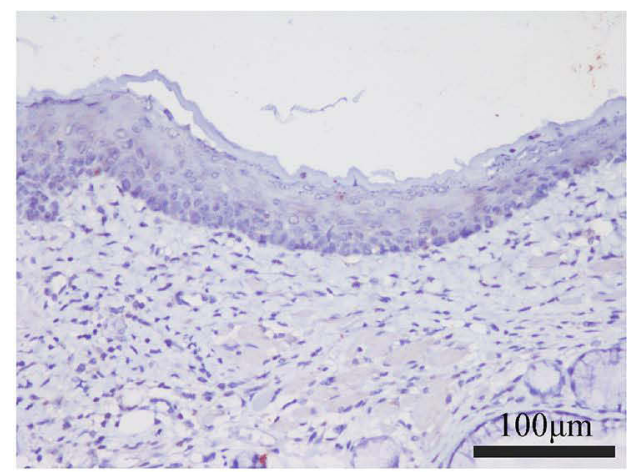

A
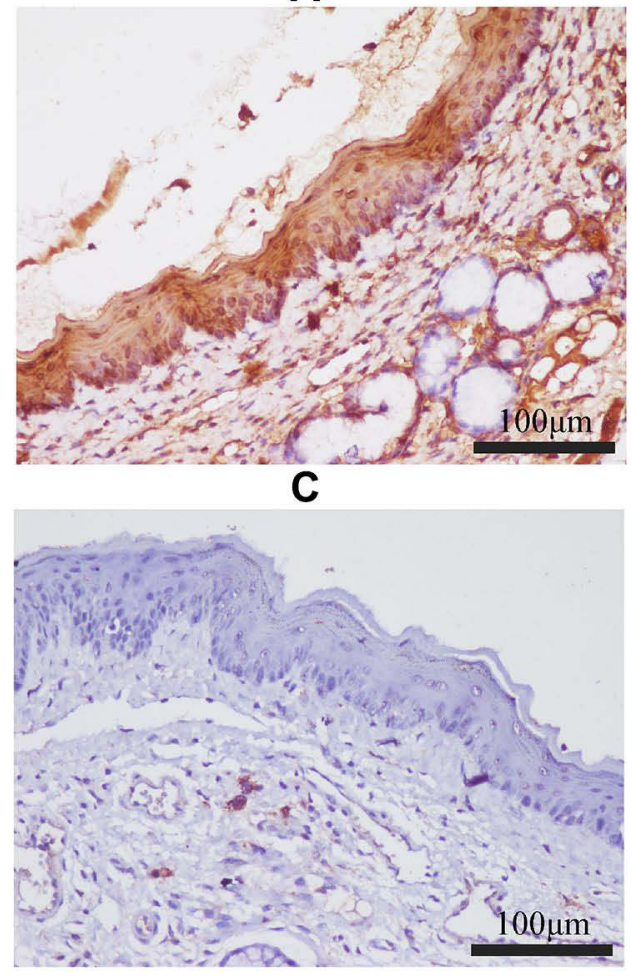

E

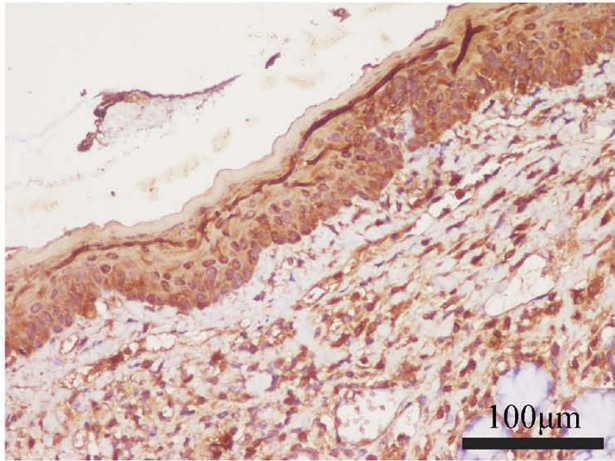

B

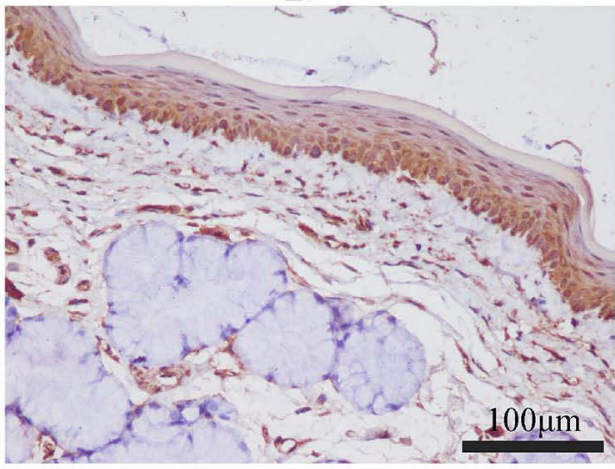

D

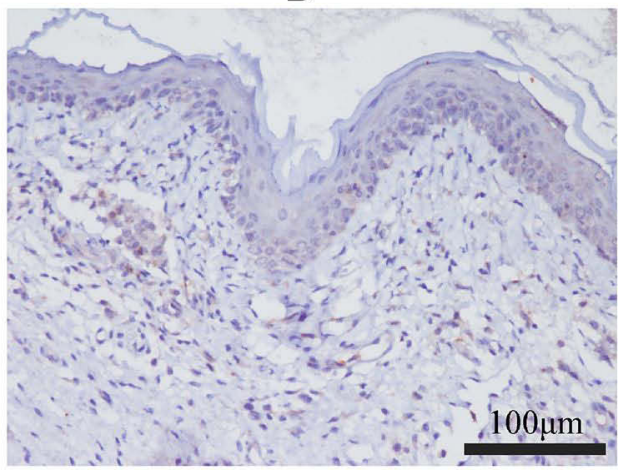

$\mathbf{F}$

Figure II The effect of FFZJF aerosol on the TLR-4 protein expression in rat's pharyngeal mucosal tissues by immunohistochemistry (400x). TLR-4 is stained brown. (A) Control group; (B) Model group; (C) FFZJF-L group; (D) FFZJF-M group; (E) FFZJF-H group; (F) AS group.

the core genes IL6, PTGS2, TLR-4, and TNF potentially targeted by FFZJF. Pathway analysis showed that these genes are involved in the regulation of classic pathways, which included regulation of cytokine secretion, inflammatory response, MyD88-dependent toll-like receptor signaling pathway, regulation of estradiol secretion, positive regulation of smooth muscle cell proliferation, negative regulation of growth of symbionts in the host, toll-like receptor signaling pathway, TNF signaling pathway, NF- $\kappa \mathrm{B}$ signaling pathway, interactions between cytokine-cytokine receptors, NOD-like receptor signaling pathway. The results suggest that FFZJF may regulate the expression of toll-like receptor signaling pathway, TNF signaling pathway, NF- $\mathrm{BB}$ signaling pathway, and other signaling pathways along with IL6, PTGS2, TLR-4, and TNF genes to achieve the purpose of treating CP.

Studies have shown that a large number of cytokines are secreted in patients with pharyngitis, including TNF$\alpha$, IL-1 $\beta$, IL6, etc. TNF- $\alpha$ is mainly produced by 


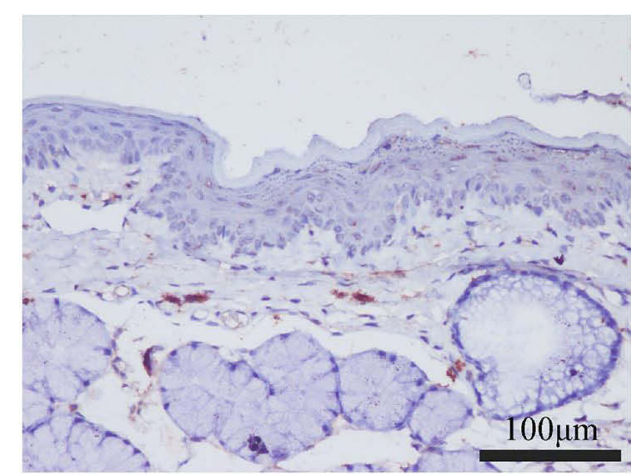

A

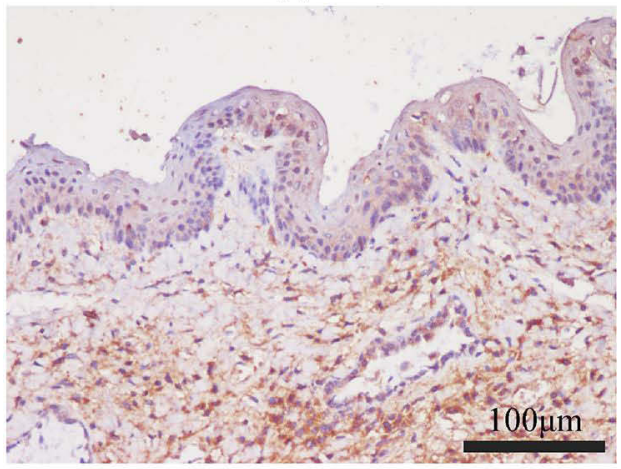

C

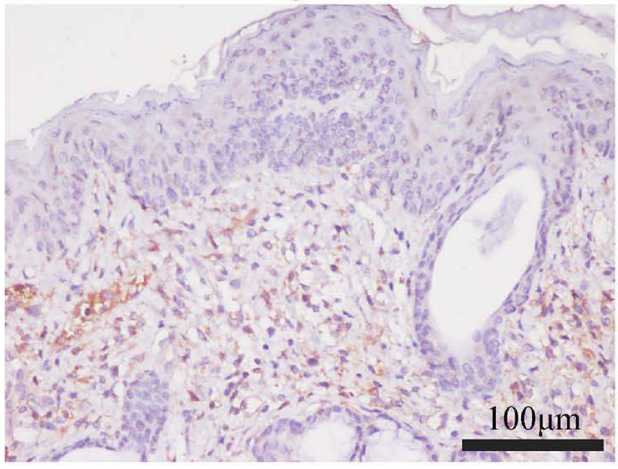

E

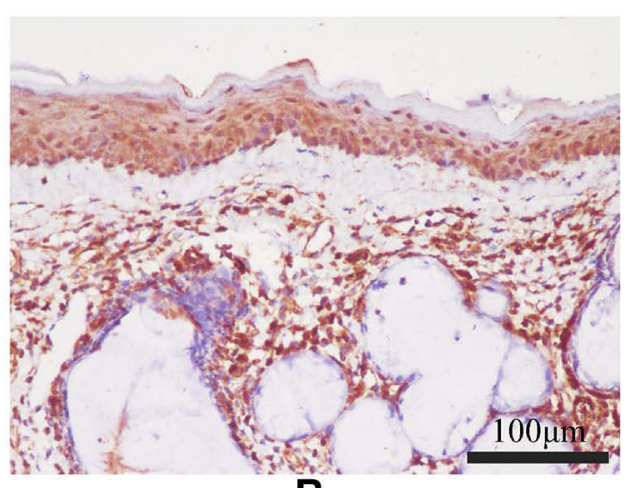

B

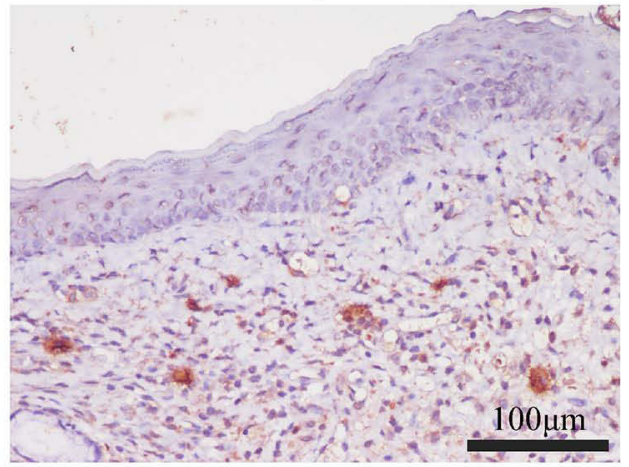

D

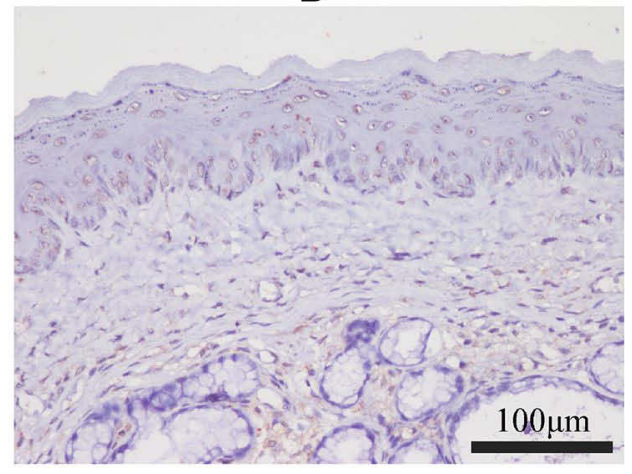

$\mathbf{F}$

Figure 12 The effect of FFZJF aerosol on the MyD88 protein expression in rat's pharyngeal mucosal tissues by immunohistochemistry (400x). MyD88 is stained brown. (A) Control group; (B) Model group; (C) FFZJF-L group; (D) FFZJF-M group; (E) FFZJF-H group; (F) AS group.

activated macrophages, NK cells, and $\mathrm{T}$ lymphocytes. IL- $1 \beta$ and IL 6 are cytokines secreted mainly by T and B lymphocytes and monocytes macrophages, which can reflect the degree of injury of inflammatory response. Cytokines in the body can interact with each other. For example, the release of IL- $1 \beta$ and TNF- $\alpha$ can encourage the occurrence of each other and cause severe reactions such as temperature rise.46 We confirm that FFZJF can down-regulate the expression of TNF- $\alpha$, IL1, $\beta$ and IL6 in serum of rats with chronic pharyngitis, and achieve the effect of treating chronic pharyngitis. NLRP3 inflammasome play a pathogenic or disease-resistant role in the development of many diseases, with upregulated expression in patients with pharyngitis.47,48 NLRP3/ASC/Caspase-1 axis induces NLRP3 inflammasome by regulating the maturation and secretion of inflammatory factors such as IL-1ßand IL-18, and activation of NLRP3 inflammasome was important to the body's resistance to invasion of exogenous pathogenic microorganisms. However, the excessive activation of NLRP3 inflammasome resulted in excessive innate immune defense against viral or bacterium with 


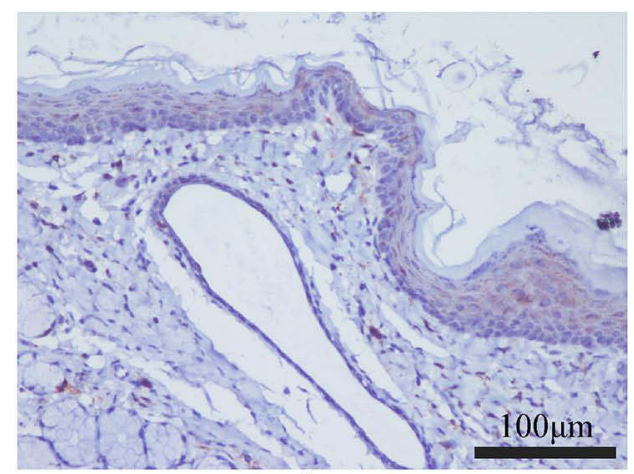

A

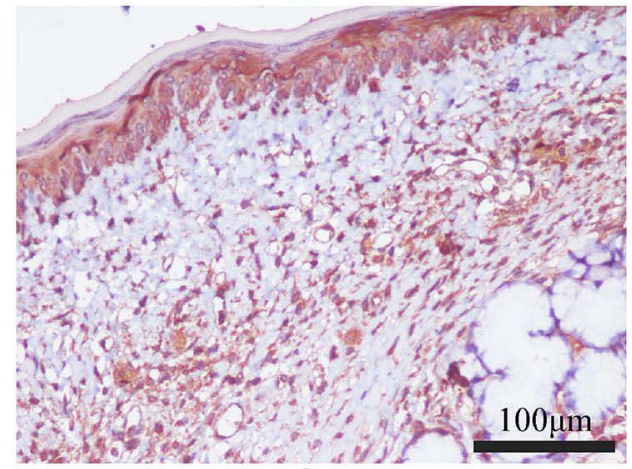

C

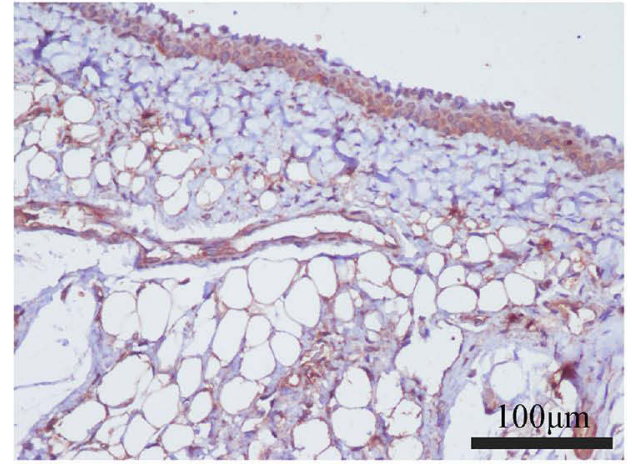

E

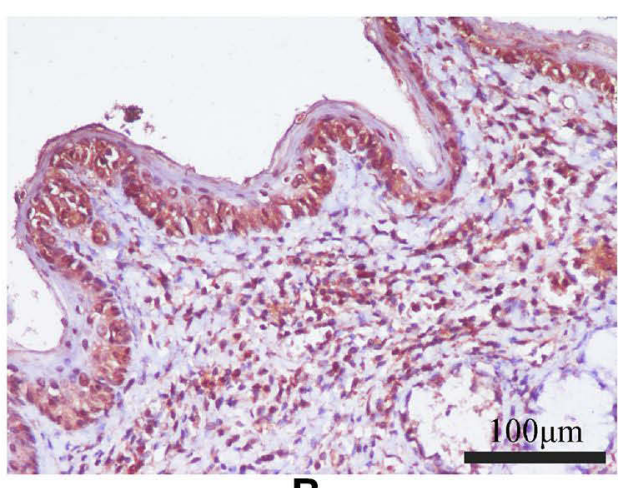

B

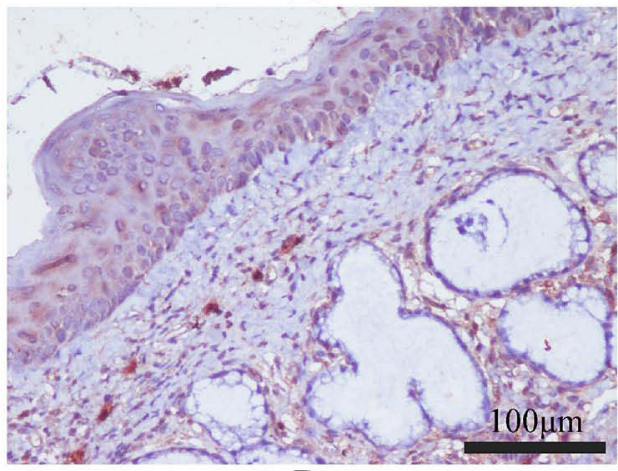

D

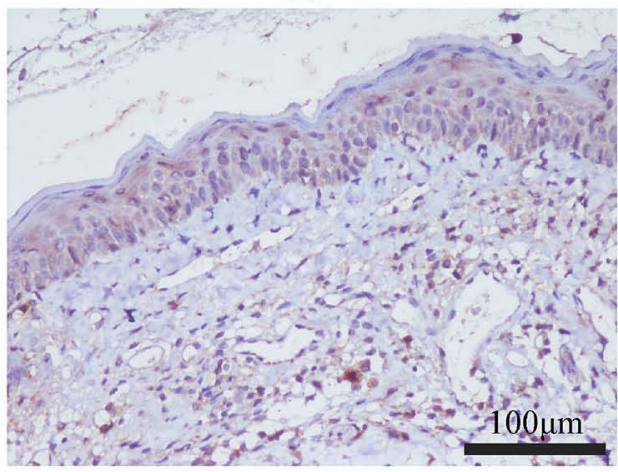

$\mathbf{F}$

Figure 13 The effect of FFZJF aerosol on the NF- $\kappa B$ P65 protein expression in rat's pharyngeal mucosal tissues by immunohistochemistry (400x). NF- $\kappa B$ P65 is stained brown. (A) Control group; (B) Model group; (C) FFZJF-L group; (D) FFZJF-M group; (E) FFZJF-H group; (F) AS group.

inflammatory cell apoptosis, tissue damage, and aggravated development of inflammatory diseases.49 In this study, network pharmacological analysis shows that FFZJF can achieve the effect of CP treatment by regulating NOD-like receptor signaling pathway, but more details about the underlying mechanism need to be further studied.

TLR-4 is a member of the toll-like receptor family. Upon pathogen recognition, TLR-4 is activated via a MyD88 (Myeloid Differentiation Primary Response Gene 88)-dependent pathway. Activated TLR-4 in turns activates NF- $\mathrm{KB}$ signaling pathway ${ }^{17}$ that eventually leads to the production and release of inflammatory cytokines (eg, TNF- $\alpha$, IL1, and IL6). ${ }^{24,45,46}$ MyD88 is an adaptor protein that plays a central role in TLR-4 signaling pathways. ${ }^{47}$ TLR-4 recognizes pathogen-associated molecular patterns (PAMPs) and dimerizes with MyD88. MyD88 signaling involves the sequential activation of IL1 receptor-associated kinases (IRAKs), adaptor molecule TNF receptor-associated factor 6 (TRAF6), and NF$\kappa \mathrm{B} .{ }^{48} \mathrm{NF}-\kappa \mathrm{B}$ activation induces the mRNA expression of inflammatory cytokines, such as TNF- $\alpha$, IL1 $\beta$, and IL6, ${ }^{49,50}$ which in turn will results in more NF- $\mathrm{BB}$ activation. Such positive feedback mechanisms elicit an inflammatory reaction cascade. With respect to TLR-4/MyD88/ $\mathrm{NF}-\kappa \mathrm{B}$ as a classical inflammatory response signaling 
Table 8 The Effect of FFZJF Aerosol on the TLR-4, MyD88, and NF- $\kappa$ B P65 Protein Expression Levels in the Rat's Pharyngeal Mucosal Tissues (Mean \pm SEM, $\mathrm{n}=8$ )

\begin{tabular}{|c|c|c|c|c|}
\hline Group & Dose (g/kg) & TLR-4 & MyD88 & NF-кB P65 \\
\hline Control & - & $1083 \pm 71$ & $1017 \pm 82$ & $1035 \pm 96$ \\
\hline CP model & - & $9161 \pm 353^{* *}$ & $9377 \pm 328 * *$ & $9139 \pm 327^{* *}$ \\
\hline FFZJF aerosol & $\begin{array}{l}2.33 \\
4.66 \\
9.32\end{array}$ & $\begin{array}{l}7882 \pm 329 * * \# \Delta \\
5854 \pm 246 * * \# \Delta \\
3750 \pm 260 * \ldots \# \Delta\end{array}$ & $\begin{array}{l}7174 \pm 360 * * \# \Delta \\
5654 \pm 329 * * \# \Delta \\
3710 \pm 203 * \ldots \#\end{array}$ & $\begin{array}{l}6427 \pm 307^{* * \# \Delta} \\
4970 \pm 340^{* * \# \Delta} \\
3768 \pm 200^{* * \#}\end{array}$ \\
\hline Ampicillin sodium & 0.93 & $3080 \pm 310^{* * \# \#}$ & $2729 \pm 201 * * \#$ & $3492 \pm 418^{* * \# \#}$ \\
\hline
\end{tabular}

Notes: As compared to treated non-CP control group: ${ }^{*} p<0.05,{ }^{*} p<<0.01$; as compared to treated CP model group: ${ }^{\#} p<0.05$, ${ }^{\#} p<0.01$; as compared to treated Ampicillin sodium group: ${ }^{\Delta} P<0.05$.

pathway, NF- $\mathrm{B}$ activation is a central link. Its activation promotes the release of inflammatory factors, which in turn promote the activation of NF- $\mathrm{KB}$ and form a positive feedback. Some studies have shown that inhibiting the expression of TLR-4 can reduce the expression of NF-kB, IL6 and improve the CP pathological changes. ${ }^{51-53}$ The etiology of $\mathrm{CP}$ is well-studied, while its mechanism is less studied. ${ }^{54}$ This study uses network pharmacology to analyze the key components, targets and signaling pathway, which lays a foundation for the study of CP pathogen and the mechanism of FFZJF treatment. In this study, the serum levels of TNF- $\alpha$, IL1 $\beta$, and IL6 in the $\mathrm{CP}$ model group were significantly higher than those in the non-CP control group. FFZJF treatment significantly reduced the serum levels of these cytokines. Histopathological examination confirmed that the inflammation was alleviated as the dose of FFZJF increased, especially in the FFZJF-H group. These results indicated that FFZJF is effective in treating CP. The significant increases of both mRNA and protein levels of TLR-4, MyD88, and NF-кB P65 in the pharyngeal mucosal tissues of the model group demonstrated that cytokines, TLR-4, MyD88, and NF- $\mathrm{BB}$ P65 are involved in the pathogenesis of $\mathrm{CP}$. We postulate that certain substances released by GAS and pharyngeal mucosal cells are recognized by TLR-4, which leads to TLR-4 MyD88, and the eventual NF- $\kappa B$ activation. As a result, the production and secretion of proinflammatory cytokines (eg, TNF- $\alpha$, IL $1 \beta$, and IL6, etc.) increase, which in turn promotes chronic inflammation, tissue repair, or tissue damages in the pharynx. Therefore, activation of the TLR-4/MyD88/NF-кB signaling pathway likely plays a critical role in the tissue
A

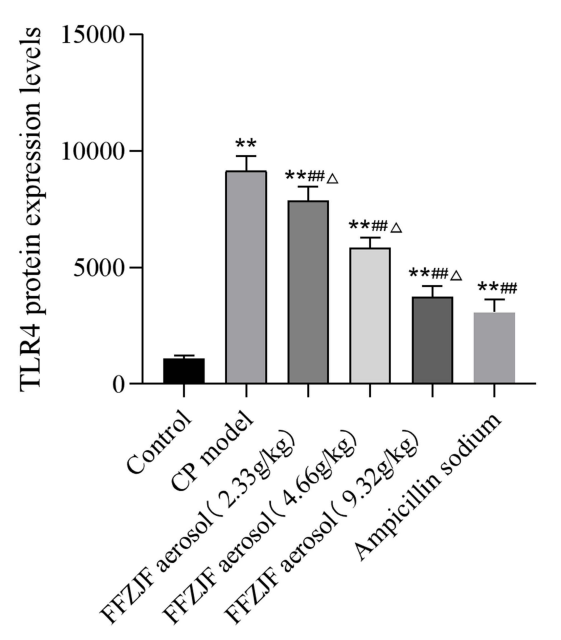

B

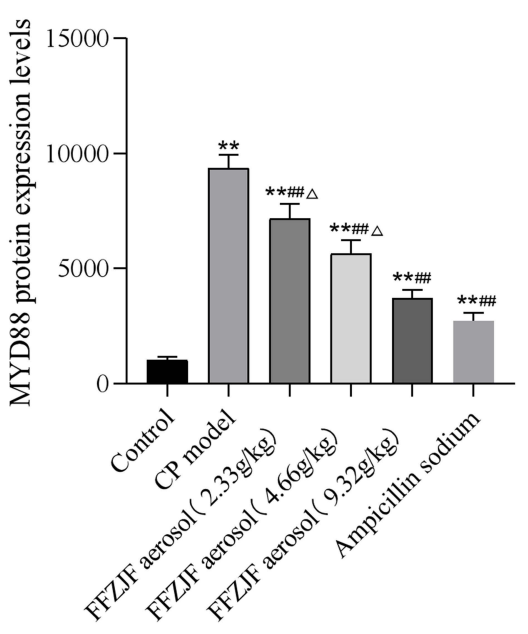

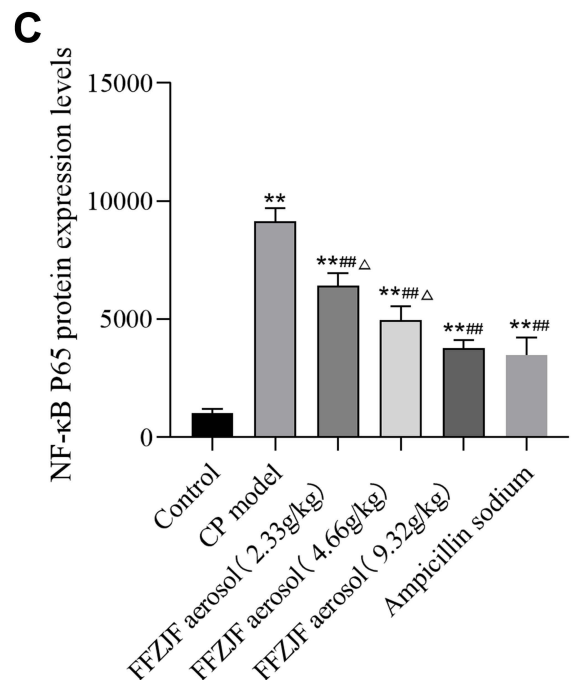

Figure 14 The effect of FFZJF aerosol on the TLR-4, MyD88 and NF- $\mathrm{KB}$ P65 protein expression in rat's pharyngeal mucosal tissues by immunohistochemistry. (A) TLR-4; (B) MyD88; (C) NF- $k$ B P65.Note: As compared to treated non-CP control group: ${ }^{*} p<0.05,{ }^{* *} p<0.0$ I; as compared to treated CP model group: ${ }^{\#} p<0.05$, ${ }^{\#} p<0.0$ I; as compared to treated Ampicillin sodium group: ${ }^{\Delta} \mathrm{P}<0.05$. 
damage observed in bacterial CP. Compared with the model group, the FFZJF-M, FFZJF-H, and AS groups had significantly decreased mRNA expression of TLR-4, MyD88, and NF-кB P65 in the pharyngeal mucosal tissues of CP animals. In all FFZJF treatment groups, the protein expression levels of TLR-4, MyD88, and NF- $\kappa$ B P65 decreased significantly. These results demonstrated that FFZJF treatment could effectively downregulate the over-

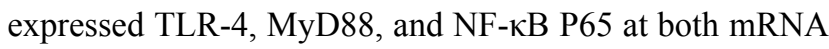
and protein levels.

\section{Conclusion}

In summary, this network pharmacology study revealed potential mechanisms of multi-component, multi-target, and multipathway effects of FFZJF in the treatment of CP. Animal experiments further validated that FFZJF treatment could not only reduce the tissue expression of TLR-4, MyD88, and NF$\kappa \mathrm{B}$ P65 at both mRNA and protein levels, but it also reduced the serum levels of TNF- $\alpha$, IL1 $\beta$, and IL6 in treated animals. These results suggest that the treatment effect of FFZJF in CP could be mediated by the reduction of cytokine levels via downregulation of TLR-4, MyD88, and NF- $\kappa \mathrm{B}$ expression. Therefore, the intervening TLR-4/MyD88/NF- $\kappa \mathrm{B}$ signaling pathway might be one of the mechanisms of FFZJF in treating CP. Our study provides a novel direction for deciphering the molecular mechanisms of FFZJF as an efficacious treatment strategy for CP. Since the pathogenesis of CP is less studied, according to the previous study, the FFZJF can be treated by anti-inflammatory characteristics $\mathrm{CP}$ and combined with the literature to select TLR-4/MyD88/NF- $\kappa \mathrm{B}$ signaling pathway as the mechanism of the phenomenon, the scope is small. Therefore, other inflammatory factors can be selected for further study.Retrieve FFZJF components in the database, some component data are still insufficient, follow up can be retrieved to ensure the accuracy of the data.

\section{Ethics Statement}

Ethics Approval and Consent to Participate:The experimental scheme was approved by the Experimental Animal Ethics Committee of Guizhou Medical University (Ethical number: 1709051) and followed the guidelines for the ethical review of laboratory animal welfare People's Republic of China National Standard GB/T 35892-2018. And the experimental process is strictly in accordance with the National Institutes of Health published guidelines for the care and use of experimental animals (NIH publication 85-23, revised in 1996) and China's guidelines for the ethical review of laboratory animal welfare.

\section{Acknowledgments}

This research was supported by the National Natural Science Foundation of China (grant 81760882 to Yanping Zhang).

Yanping Zhang and Taohua Yuan contributed equally to this paper and should be considered as the first authors.

\section{Disclosure}

The authors report no conflicts of interest in this work.

\section{References}

1. Liu F, Wu BL, Chen T, He JJ. Survey on the oral health related quality of life in patients with chronic pharyngitis. $J$ Dent Prev Treat. 2016;24:244-247.

2. Chen YH, Luo R, Lei SS, et al. Anti-inflammatory effect of Ganluyin, a Chinese classic prescription, in chronic pharyngitis rat model. BMC Complement Med Ther. 2020;20:265. doi:10.1186/ s12906-020-03057-5

3. Leone CA, Caruso AA, Allocca V, Barra E, Leone R. Pilot study on the effects of high molecular weight sodium hyaluronate in the treatment of chronic pharyngitis. Int J Immunopathol Pharmacol. 2015;28:532-538. doi:10.1177/0394632015586497

4. Fan MY, Zhang XY, Zhao X, et al. Network meta-analysis of efficacy and safety of classic prescription and Chinese patent medicine in treatment of chronic pharyngitis. Drug Eval Res. 2021;44 (04):856-868

5. Soderholm AT, Barnett TC, Sweet MJ, Walker MJ. Group A streptococcal pharyngitis: immune responses involved in bacterial clearance and GAS-associated immunopathologies. J Leukocyte Biol. 2018;103:193-213. doi:10.1189/jlb.4MR0617-227RR

6. Pan GF, Cheng L, Liu Y, Feng XZ. Meta-analysis of Yangyin Qingfei decoction and Yangyin Qingfei oral liquid in the treatment of chronic pharyngitis. China J Tradit Chin Med Pharm. 2018;33:2360-2363.

7. Lu C, Song Y, Zhang J, et al. Yanshu spraying agent, a traditional Chinese medicine, relieves chronic pharyngitis in animals by anti-inflammatory and antibacterial effects. Exp Ther Med. 2014;7:990-994. doi:10.3892/etm.2014.1524

8. Gu W, Bi B, Jin X, Chang Y, Kong Y. Clinical observation of chronic pharyngitis treated with acupuncture. J Guangzhou Univ Tradit Chin Med. 2016;33:38-42.

9. Liao LY, Zhang YP. Research progress on etiology and pathogenesis of chronic pharyngitis. J Audiol Speech Pathol. 2019;51 (03):210-212.

10. Liao LY, Zhang YP, Xu XM. Clinical obervation on compound zhonjiefeng aerosol inhalation combined with acupoint injection at lianquan, cricothyroid membrane and tiantu on acute pharyngitis of lung -stomach hyperthermia. JETCM. 2019;28(2):256-258.

11. Li YS, Zhang YP. A randomized controlled study of compound zhongjiefeng atomization in the treatment of chronic pharyngitis. Gen J Stomatol. 2018;5(33):190-191.

12. Centor RM, Atkinson TP, Ratliff AE, et al. The clinical presentation of fusobacterium-positive and streptococcal-positive pharyngitis in a university health clinic. Ann Intern Med. 2015;162:241-247. doi:10.7326/M14-1305

13. Oliver J, Wadu EM, Pierse N, Moreland NJ, Williamson DA, Baker MG. Group A Streptococcus pharyngitis and pharyngeal carriage: a meta-analysis. PLoS NeglTrop Dis. 2018;12:e0006335. doi:10.1371/journal.pntd.0006335

14. Xianmei X, Zhang Y, Yongyue GA. A preliminary investigation on anti-inflammatory and antibacterial effect of compound sarcandra aerosol. Agric Sci Technol. 2016;17(6). 
15. Li S, Zhang B. Traditional Chinese medicine network pharmacology: theory, methodology and application. Chin J Nat Med. 2013;11 (2):0110-0120. doi:10.1016/S1875-5364(13)60037-0

16. Zhou Z, Chen B, Chen S, et al. Applications of network pharmacology in traditional Chinese medicine research. Evid Based Complement Alternat Med. 2020;2020:1646905. doi:10.1155/2020/ 1646905

17. Newman DJ. Modern traditional Chinese medicine: identifying, defining and usage of TCM components. Adv Pharmacol. 2020;87:113-158.

18. Ru J, Li P, Wang J, et al. TCMSP: a database of systems pharmacology for drug discovery from herbal medicines. J Cheminf. 2014;6:13. doi:10.1186/1758-2946-6-13

19. Shanghai Institute of Organic Chemistry, Chinese Academy of Sciences. Chemical professional database[DB/OL]. 1978-2016. Available from: http://www.organchem.csdb.cn. Accessed June 18, 2021.

20. Zhu N, Hou J. Exploring the mechanism of action Xianlingubao Prescription in the treatment of osteoporosis by network pharmacology. Comput Biol Chem. 2020;85:107240. doi:10.1016/j. compbiolchem.2020.107240

21. Yang X, Li Y, Lv R, Qian H, Chen X, Yang CF. Study on the multitarget mechanism and key active ingredients of herba siegesbeckiae and volatile oil against rheumatoid arthritis based on network pharmacology. Evid Based Complement Alternat Med. 2019;2019:8957245. doi:10.1155/2019/8957245

22. Qin TY, Wu LL, Hua Q, et al. Prediction of the mechanisms of action of Shenkang in chronic kidney disease: a network pharmacology study and experimental validation. $J$ Ethnopharmacol. 2020;246:112128. doi:10.1016/j.jep.2019.112128

23. Xu Y, Chen WY, Chen ZR, et al. Mechanism of action of Xiaoyao San in treatment of ischemic stroke is related to anti-apoptosis and activation of PI3K/Akt pathway. Drug Des Devel Ther. 2021;15:753-767. doi:10.2147/DDDT.S280217

24. Byrd-Leifer CA, Block EF, Takeda K, Akira S, Ding A. The role of MyD88 and TLR-4 in the LPS-mimetic activity of Taxol. Eur $J$ Immunol. 2001;31:2448-2457. doi:10.1002/1521-4141(200108) 31:8<2448::AID-IMMU2448>3.0.CO;2-N

25. Wang F, Wang L, Liu F, et al. Investigation of the mechanism of the reduction of anthracycline-induced cardiotoxicity by Qishen Huanwu capsule based on network pharmacology. Ann Palliat Med. 2021;10 (1):16-28. doi:10.21037/apm-20-2204

26. Hu SH, Dan WC, Liu JL, et al. Use of traditional Chinese medicine in relieving EGFR-TKI-associated diarrhea based on network pharmacology and data mining. Evid Based Complement Alternat Med. 2021;2021:5530898. doi:10.1155/2021/5530898

27. Wang SJ, Gong XF, Feng JL, et al. Study on the mechanism of Jiawei Shengjiang powder in improving male asthma-induced asthenospermia based on network pharmacology and bioinformatics. Drug Des Devel Ther. 2021;15:1245-1259. doi:10.2147/DDDT.S296901

28. Liang L Investigate the effects of a new formula on acute and chronic pharyngitis. Shanxi: Shanxi University; 2012.

29. Peng SL, Zhong Q, Xiong DJ, Shi JP, Zhou Y, Li X. Study on animal models of chronic pharyngitis. J Chengdu Univ Tradit Chin Med. 1999;22:51-52.

30. Li YK. Experimental Methodology of Traditional Chinese Medicine Pharmacology. Shanghai: Shanghai Scientific and Technical Publishers; 2006:38-39.

31. Pereira Beserra F, Sérgio Gushiken LF, Vieira AJ, et al. From inflammation to cutaneous repair: topical application of lupeol improves skin wound healing in rats by modulating the cytokine levels, NF- $\mathrm{kB}$, $\mathrm{Ki}-67$, growth factor expression, and distribution of collagen fibers. Int J Mol Sc. 2020;21(14):4952. doi:10.3390/ijms21144952

32. Zhai W, Yao M, Chen J. Comparative study of lymphoid follicles in mucosa of pharynx and mucosal associated lymphoid tissues in paranasal sinuses. J Clin Otorhinolaryngol Head Neck Surg. 2013;27:806-808.
33. Zeng YL, Liu JY, Zhang Q, et al. Sarcandra glabraThe traditional uses, phytochemistry and pharmacology of (Thunb.) Nakai, a Chinese herb with potential for development: review. Front Pharmacol. 2021;12:652926. doi:10.3389/fphar.2021.652926

34. Lewin G, Joel M, Peter B, et al. Food grade safflower concentrate: no evidence for reproduction and early developmental toxicity. Reprod Toxicol. 2021;21:S0890-6238.

35. Song ZW, Xiang XJ, Li JH, et al. Ruscogenin induces ferroptosis in pancreatic cancer cells. Oncol Rep. 2020;43:516-524. doi:10.3892/ or.2019.7425

36. Jung JA, JhH N, Jang MS, et al. Safety evaluation of fermented Platycodon grandiflorus (Jacq.) A.DC. extract: genotoxicity, acute toxicity, and 13-week subchronic toxicity study in rats. J Ethnopharmacol. 2021;275:114138. doi:10.1016/j.jep.2021.114138

37. Li WF, Fan T, Zhang YM, et al. Houttuynia cordata Thunb. volatile oil exhibited anti-inflammatory effects in vivo and inhibited nitric oxide and tumor necrosis factor-aproduction in LPS-stimulated mouse peritoneal macrophages in vitro. Phytother Res. 2013;27:1629-1639. doi:10.1002/ptr.4905

38. Liu T, Han SY. Optimization and anti-chronic pharyngitis of luteolin, baicalein and quercetin monomer. Sci Technol Vis. 2015;09:22+84.

39. Liu ZY, Yao XQ, Sun BH, et al. Pretreatment with kaempferol attenuates microglia-mediate neuroinflammation by inhibiting MAPKs-NF- $\mathrm{KB}$ signaling pathway and pyroptosis after secondary spinal cord injury. Free Radic Biol Med. 2021;168:142-154. doi:10.1016/j.freeradbiomed.2021.03.037

40. Liu T, Chen S. Sarcandra glabra combined with lycopene protect rats from lipopolysaccharide induced acute lung injury via reducing inflammatory response. Biomed Pharmacother. 2016;84:34-41. doi:10.1016/j.biopha.2016.09.009

41. Chun JM, Nho KJ, Kim HS, et al. An ethyl acetate fraction derived from Houttuynia cordata extract inhibits the production of inflammatory markers by suppressing NF-KB and MAPK activation in lipopolysaccharide-stimulated RAW 264.7 macrophages. BMC Complement Altern Med. 2014;14(1):234. doi:10.1186/1472-6882$14-234$

42. Wang H, Liu J, Yang Y, et al. Hydroxy-Safflower Yellow A inhibits the TNFR1-mediated classical pathway by inducing shedding of TNFR1. Phytother Res. 2016;30(5):790-796. doi: $10.1002 /$ ptr.5579

43. Qu Y, Zhou L, Wang C. Effects of platycodin D on IL1 $\beta$-induced inflammatory response in human osteoarthritis chondrocytes. Int Immunopharmacol. 2016;40:474-479. doi:10.1016/j.intimp.2016. 09.025

44. Zhang YP, Wu N, Shi YH. Clinical observation of added nebulizer of Fufang Zhongiiefeng aerosol in treating 40 cases with gastroesophageal reflux laryngitis. Chin J Exp Tradt Med Formulae. 2015;21 (02):209-212.

45. Bowie AG, Haga IR. The role of Toll-like receptors in the host response to viruses. Mol Immunol. 2005;42:859-867. doi:10.1016/j. molimm.2004.11.007

46. Wu L, Du L, Ju Q, et al. Silencing TLR-4/MyD88/NF-кB signaling pathway alleviated inflammation of corneal epithelial cells infected by ISE. Inflammation. 2020;1-12.

47. Takeda K, Akira S. Microbial recognition by Toll-like receptors. J Dermatol Sci. 2004;34:73-82. doi:10.1016/j.jdermsci.2003.10.002

48. Perkins ND. Post-translational modifications regulating the activity and function of the nuclear factor kappa B pathway. Oncogene. 2006;25:6717-6730. doi:10.1038/sj.onc. 1209937

49. Gratz N, Siller M, Schaljo B, et al. Group A streptococcus activates type I interferon production and MyD88-dependent signaling without involvement of TLR2, TLR-4, and TLR9. J Biol Chem. 2008;283:19879-19887. doi:10.1074/jbc.M802848200

50. Giridharan S, Srinivasan M. Mechanisms of NF-кB p65 and strategies for therapeutic manipulation. J Inflamm Res. 2018;11:407-419. doi:10.2147/JIR.S140188 
51. $\mathrm{Xu} \mathrm{JH}$. To investigate the mechansim of treating acute pharyngitis with Zizheng Dihuang decoction based on TLR-4/NF- $\mathrm{kB}$ signaling pathway. Anhui University of Chinese Medicine; 2020.

52. Wu N, Huang YX, Shi X, et al. Effect of lysionotus pauciflorus maxim on signaling pathway of TLR-4-MyD88-NF- $\mathrm{BB}$ in lung tissue of rats with chronic bronchitis. J Guizhou Med Univ. 2020;45 (11):1283-1288.

53. Zhang J, Xiao F, Zhang LL, et al. Alpha-lipoic acid preconditioning and ischaemic postconditioning synergistically protect rats from cerebral injury induced by ischemia and reperfusion partly via inhibition TLR-4/MyD88/ NF- $\mathrm{BB}$ signaling pathway. Cell Physiol Biochem. 2018;51(3):1448-1460. doi:10.1159/000495593
54. Chen QB, Wang Y, Li F, et al. Research progress on etiology and pathogenesis of chronic pharyngitis. J Audiol Speech Pathol. 2019;27 (02):224-228.

\section{Publish your work in this journal}

Drug Design, Development and Therapy is an international, peerreviewed open-access journal that spans the spectrum of drug design and development through to clinical applications. Clinical outcomes, patient safety, and programs for the development and effective, safe, and sustained use of medicines are a feature of the journal, which has also been accepted for indexing on PubMed Central. The manuscript management system is completely online and includes a very quick and fair peer-review system, which is all easy to use. Visit http://www. dovepress.com/testimonials.php to read real quotes from published authors.

Submit your manuscript here: https://www.dovepress.com/drug-design-development-and-therapy-journal 